\title{
On the Importance of Baseline Setting in Carbon Offsets Markets
}

Antonio Bento, Ravi Kanbur, and Benjamin Leard

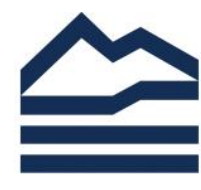

RESOURCES FOR THE FUTURE 


\title{
On the importance of baseline setting in carbon offsets markets
}

\author{
Antonio Bento, Ravi Kanbur and Benjamin Leard*
}

\begin{abstract}
Incorporating carbon offsets in the design of cap-and-trade programs remains a controversial issue because of its potential unintended impacts on emissions. At the heart of this discussion is the issue of crediting of emissions reductions. Projects can be correctly, over- or under-credited for their actual emissions reductions. We develop a unified framework that considers the supply of offsets within a cap-and-trade program that allows us to compare the relative impact of over-credited offsets and under-credited emissions reductions on overall emissions under different levels of baseline stringency and carbon prices. In the context of a national carbon pricing scheme that includes offsets, we find that the emissions impacts of over-credited offsets can be fully balanced out by under-credited emissions reductions without sacrificing a significant portion of the overall supply of offsets, provided emissions baselines are stringent enough. In the presence of high predicted business-as-usual (BAU) emissions uncertainty or low carbon prices, to maintain the environmental integrity of the program, baselines need to be set at stringent levels, in some cases below 50 percent of predicted BAU emissions. As predicted BAU emissions uncertainty declines or as the carbon market achieves higher equilibrium prices, however, less stringent baselines can balance out the emissions impacts of over-credited offsets and under-credited emissions reductions. These results imply that to maintain environmental integrity of offsets programs, baseline stringency should be tailored to project characteristics and market conditions that influence the proportion of over-credited offsets to under-credited emissions reductions.
\end{abstract}

Keywords: Carbon offsets, crediting, environmental integrity

\footnotetext{
*Bento: University of Southern California, Sol Price School of Public Policy and NBER, Ralph and Goldy Lewis Hall 214, Los Angeles, CA 90089 (e-mail: abento@usc.edu). Kanbur: Cornell University, 301-J Warren Hall, Ithaca, NY 14853 (e-mail: sk145@cornell.edu). Leard: Resources for the Future, 1616 P St. NW, Washington D.C. 20036 (e-mail: leard@rff.org).
} 


\section{Introduction}

Complementing cap-and-trade programs with carbon offsets supplied from uncapped sectors is recognized as a way of achieving emissions reduction targets at lower economic cost (Basu, 2009; Brown and Adger, 1994; Chameides and Oppenheimer, 2007; Lehmann, 2007; Victor, 2012). However, awarding offsets to projects requires the setting of a baseline that reflects the project's BAU emissions. Offsets are counted based on documented emissions relative to baselines. If the offsets project managers have more information on the project's BAU emissions than the regulator that assigns the project baseline, then the program may attract projects that have baselines above their BAU emissions. Managers opt these projects into the program and can claim offsets up to their baseline while not reducing emissions (Fischer, 2005; Menges, 2003; Meyers, 1999; Rentz, 1998). When these offsets are sold to firms regulated under a cap-and-trade program, overall emissions can increase (Gillenwater et al., 2007; Kintisch, 2008; Maslin, 2011; Schneider, 2009a; Zhang and Wang, 2011). Studies have documented this issue of asymmetric information in various contexts, including $\mathrm{SO}_{2}$ tradable permit markets (Montero, 1999, 2000), incentives to reduce emissions from deforestation (Busch et al., 2012; Van Benthem and Kerr, 2013), design payments for environmental services (Ferraro, 2008) and sectoral crediting of voluntary emissions reductions (Millard-Ball, 2013). Other studies have suggested various solutions for this problem, including using multiple policy instruments (Bento et al., 2015; Calvin et al., 2015; Horowitz and Just, 2013) and contract design (Mason and Plantinga, 2013).

The issue at hand is one of crediting of emissions reductions. A program may award a project with offsets that exceed the project's emissions reductions, leading to the production of offsets that we define as over-credited offsets. But the crediting system may also lead to emissions reductions that do not generate offsets. This happens when an opted in project is assigned a baseline below its BAU emissions. These projects lower emissions more than the quantity of offsets they earn and can reduce aggregate emissions by the difference between the project's baseline and its predicted BAU emissions. We call the reduction in aggregate emissions under-credited emissions reductions.

Such reductions have been identified as a source that can counteract the emissions consequences of overcredited offsets. Schneider (2009b) suggests that setting baselines below BAU emissions can lead to an atmospheric benefit as offsets projects are credited with fewer offsets than their true emissions reductions. Gillenwater (2012a,b) correctly isolates the impact of over-crediting projects and awarding offsets to projects that are non-additional. In both cases, aggregate emissions can increase. Bento et al. (2015), Erickson et al. (2014) and Warnecke et al. (2014) model under- and over-crediting of offsets projects and find that undercrediting can play a significant role in maintaining environmental integrity of climate mitigation programs.

Even with these studies, however, little is known about the relative importance of over-credited offsets 
and under-credited emissions reductions and how the relative magnitudes of each quantity vary in response to key market parameters, including the carbon price, uncertainty in BAU emissions and mitigation costs. We quantify the relative magnitudes of the two emissions impacts under a wide range of market parameters by calibrating a simple model of carbon abatement in the United States that accounts for adverse selection among offsets projects (Bento et al., 2015; Bushnell, 2012). Therefore we are able to identify policies that may balance these two quantities when facing different market conditions. We find that the emissions impacts of over-credited offsets can be fully balanced out by under-credited emissions reductions without sacrificing a significant portion of the overall supply of offsets, provided emissions baselines are stringent enough. When predicted BAU emissions uncertainty is low or as the carbon market achieves high equilibrium prices, less stringent baselines are required to balance out the emissions impacts of over-credited offsets and under-credited emissions reductions. Our results suggest that to maintain environmental integrity of carbon offsets programs without sacrificing substantial cost savings from these programs, policy makers should tailor baseline stringency to project characteristics and market conditions that influence the proportion of over-credited offsets to under-credited emissions reductions.

\section{Methods}

Our model includes an uncapped sector that comprises heterogeneous projects and a capped sector represented by a single cost-minimizing firm. Offsets are supplied by projects in the uncapped sector. For each capped sector reduction target that we consider, we assume that a quantity of emissions permits is grandfathered to the regulated firms that equals regulated firm BAU emissions minus the reduction target. While others have pointed to other allocation methods for these types of systems (Goulder et al., 2010), whether permits are grandfathered or auctioned does not change our conclusions but instead influences the distribution of rents among firms and the regulator. The capped sector complies with the program by holding permits, reducing emissions through abatement or buying offsets. Permit and offset prices are solved endogenously so that the demand for permits and offsets by capped firms equals the supply of permits by the regulator and the supply of offsets from projects, respectively.

In equilibrium, the offsets price equals the permit price without additional distortions, such as offsets usage limits, trade ratios or offsets usage transaction costs. In some cap-and-trade programs, offsets sell for a lower price than permits, possibly due to the distortions mentioned (Braun et al., 2015; Mansanet-Bataller et al., 2011; Naegele, 2015). We do not model a gap between permits and offsets for the sake of consistency with previous analyses of Waxman-Markey (Kile, 2009). Instead, we model transaction costs on the offsets supply side, which has similar qualitative effects to a demand side transaction cost. 


\section{The Supply of Offsets}

Offsets supply is derived from profit maximization behavior of offsets project managers. We model the managerial decisions of projects to supply offsets through a project-specific profit function:

$$
\pi_{i}=\max _{s_{i} \leq e_{i} \leq u_{i}}\left\{\left(p-t_{i}\right)\left(b_{i}-e_{i}\right)-c_{i}\left(u_{i}-e_{i}\right)\right\}
$$

Supply decisions by project managers are based on six variables: BAU emissions $\left(u_{i}\right)$, sequestration potential $\left(s_{i}\right)$, a marginal cost of mitigation $\left(c_{i}\right)$, an assigned emissions baseline $\left(b_{i}\right)$, a per unit transaction cost $\left(t_{i}\right)$ and an equilibrium price of offsets that is common to all projects $(p)$. The manager of project $i$ knows with certainty its project's BAU emissions, while the regulator only knows predicted BAU emissions, which equal project-specific BAU emissions plus a project-specific emissions shock. Baselines are set as a function of predicted BAU emissions. Ex-post emissions are assumed to be common knowledge that the policy maker can perfectly observe. The emissions shocks are independently and identically drawn from a normal distribution.

Each manager's decision whether to opt in its project and whether to mitigate is based on equation (1). Project managers compare the profits of the difference decisions and choose the combination that solves the problem stated in equation (1). In the Supplementary Material we analytically derive optimal choices from equation (1) and divide approved projects into different categories based on project characteristics. We summarize this categorization with Figure 1. Project $i$ 's BAU emissions, $u_{i}$, is shown on the horizontal axis while its marginal costs of mitigation, $c_{i}$, is on the vertical axis.

The manager of an approved project can either commence with the project (i.e., opts into the program) or decide not to start the project (i.e., does not opt in). Approved projects that have either high marginal costs of mitigation or relatively low baselines are not profitable enough for the manager to opt in. These are designated by the purple and blue cross-hashed regions in Figure 1. There are some projects that are profitable enough for the manager to opt in and have its project perform mitigation but are under-credited because they are assigned a baseline below their BAU emissions. These are projects that fall into the green region and are characterized by marginal costs of mitigation that are sufficiently below the offsets price less transaction costs. Managers of projects that are assigned a baseline above the project's BAU emissions opt in their projects and are over-credited. These projects fall into the red region of Figure 1 and would have commenced without the program taking place. This is because these projects have marginal costs of mitigation above the offsets price less transaction costs. The orange region in Figure 1 includes projects that perform mitigation but are over-credited. These projects would not have occurred in the absence of the program, since their marginal costs of mitigation fall below the offsets price less transaction costs. However, 
they are awarded a greater quantity of offsets than the quantity of emissions reductions they provide. In this case, the projects earn some offsets that correspond to mitigation and some that do not correspond to mitigation (e.g., over-credited offsets). When regulated sectors under a cap-and-trade program can use offsets to meet the cap, the supply of over-credited offsets leads to overall emissions increases while under-credited emissions reductions lead to overall emissions reductions.

Managerial decisions yield offsets supply and under-credited emissions reductions, which are used to calculate the change in emissions (see Supplementary Equation 17). We exclude a supply of international offsets in our benchmark simulations because of the high level of uncertainty in existing estimates for this supply. Our sensitivity analysis, however, includes scenarios that represent a program that includes international offsets supply (see Supplementary Tables 7, 8 and 9).

While under-credited emissions reductions are affected by the price of offsets, over-credited offsets are not. As long as the offsets price less transaction cost is positive, over-credited projects are profitable and are opted in. Thus, increasing the offset price has no effect on the supply of over-credited offsets for sufficiently high price levels. In sharp contrast, the opt-in decision of an under-credited project depends on the offset price. As the offset price increases, more under-credited projects become profitable, represented by an expansion up of the lower-right green region in Figure 1. See the Supplementary Material for a graphical illustration of this effect.

\section{The Capped Sector}

Offsets are supplied to the capped sector that must comply with an emissions reduction target. We model the capped sector as a representative firm, an assumption that is consistent with prior literature (Fell et al., 2012; Fell and Morgenstern, 2010). We calibrate the abatement cost structure of the capped sector with processed simulation output from the EPA's analysis of the Waxman-Markey bill (EPA, 2009c). The capped sector emissions reduction target translates into a fixed supply of emissions permits. The capped sector must hold one emissions permit or one offset for every unit of emissions that it does not mitigate.

\section{Equilibrium}

Equilibrium is determined by equating permit supply and demand and offsets supply and demand. Permit and offset prices are determined endogenously through these market-clearing conditions. This equilibrium is static as we do not model dynamic decisions of capped sector firms or offsets projects. Dynamic capand-trade models, however, typically find that with unconstrained banking and borrowing and increasing reduction target stringency, equilibrium prices increase at the rate of interest over time (EPA, 2009b; Rubin, 1996). This result is consistent with how we frame our scenarios where the lowest prices emerge early in the program under low capped sector reduction targets and the highest prices come later in the program under stringent targets. 


\section{Calibration}

We calibrate the model to represent a stylized federal cap-and-trade program in the United States. We assign values of the mitigation cost parameters based on estimates used in the EPA's analysis of WaxmanMarkey (EPA, 2009a,c). We base our simulation on Waxman-Markey parameters because this bill is the most prominent federal legislation in the United States to include an offsets provision and therefore remains the most representative offsets policy that the United States may adopt in the future.

We calibrate the distribution of predicted BAU emissions that yields an expected quantity of over-credited offsets equal to 30 percent of total offsets supply when baselines are set to equal predicted BAU emissions in an equilibrium with a carbon price of $\$ 25$ per ton of $\mathrm{CO}_{2} e$. We calibrate a domestic offsets supply function to data on mitigation costs from forecasts of mitigation cost curves from various offsets supply sources in the United States, including livestock management, crop management, afforestation, forest management and soil sequestration (EPA, 2009d). For full details of the model structure and calibration, see the Supplementary Material.

\section{Results}

We discovered that for a range of parameter values, under-credited emissions reductions exceed the supply of over-credited offsets if baselines are set stringent enough. Figure 2 shows the composition of offsets and emissions changes for a range of baselines on the horizontal axis, expressed as a proportion of predicted BAU emissions. A proportion less than one implies that every project's baseline is less than its predicted BAU emissions. The vertical axis measures offsets supply and emissions changes in terms of million metric tons of $\mathrm{CO}_{2}$ equivalent $\left(\mathrm{MMTCO}_{2} \mathrm{e}\right)$.

We present outcomes under nine combinations of predicted BAU emissions uncertainty and reduction target stringency. We allow both market attributes to vary from low, to medium to high, where the medium level of each attribute is our benchmark. Our benchmark medium reduction target represents a mediumrun abatement target of 2,000 $\mathrm{MMTCO}_{2} \mathrm{e}$, which was scheduled under Waxman-Markey legislation to be achieved by 2026 (EPA, 2009a). The low and high cases represent short- and long-run reduction targets under the same legislation, respectively. The low and high cases for predicted BAU emissions uncertainty represent less and more uncertainty on predicting BAU emissions around the benchmark level of uncertainty, respectively.

The different curves show outcomes for the supply of over-credited offsets $(O C O)$, aggregate change in emissions $(\Delta E)$, and under-credited emissions reductions $(U C E R)$. The aggregate change in emissions is relative to a program that does not include offsets. If the capped sector reduction target is high and 
when baselines are set to be less than about 65 percent predicted BAU emissions, under-credited emissions reductions exceed the supply of over-credited offsets for all considered levels of predicted BAU emissions uncertainty (Figure 2g,h,i). In particular, when BAU emissions uncertainty is low, baselines set below 80 percent of predicted BAU emissions achieve a similar result. Under these scenarios and for this range of baselines, emissions decrease. A high reduction target yields a higher equilibrium offsets price, which encourages greater participation by project developers as the marginal returns to mitigating emissions is higher. Therefore it is more likely for managers of projects with assigned baselines less than their BAU emissions to opt in. This increases the quantity of under-credited emissions reductions while having no effect on the supply of over-credited offsets. When the degree of uncertainty on BAU emissions is low (Figure 2g), less stringent baselines are necessary for aggregate emissions to fall. Low BAU emissions uncertainty implies that a project is more likely to receive a baseline that matches its BAU emissions. This has the effect of reducing the supply of over-credited offsets since there will be fewer projects that have baselines above their BAU emissions.

If the degree of uncertainty for predicted BAU emissions is high, it is less likely for the quantity of undercredited emissions reductions to exceed the supply of over-credited offsets (Figure 2c,f,i). A higher degree of uncertainty implies that projects have more extreme predicted BAU emissions. A project that has predicted BAU emissions that are substantially larger than its BAU emissions is more likely to receive a baseline that exceeds its BAU emissions. The manager of this project will likely opt in and earn over-credited offsets. On the other hand, a project that has predicted BAU emissions that are substantially lower than its BAU emissions is more likely to receive a baseline so low that its manager will no longer find it profitable to opt in its project. In this case, the project does not generate under-credited emissions reductions. When the capped sector reduction target is low (Figure 2c), this effect is amplified as project managers have a lower revenue incentive to opt in their project and have it mitigate emissions. In this case, project baselines must be very stringent - less than 35 percent of predicted BAU emissions - for the quantity of under-credited emissions reductions to exceed the supply of over-credited offsets. For a capped sector reduction target of 2,000 $\mathrm{MMTCO}_{2} \mathrm{e}$ and the benchmark level of uncertainty (Figure 2e), the net effect on emissions of creating an offsets market is zero when baselines equal 70 percent of predicted BAU emissions.

Our analysis thus far suggests that the emissions consequences of under-credited emissions reductions can potentially cancel the emissions consequences from the supply of over-credited offsets if baselines are stringent. Setting baselines low, however, may eliminate a significant supply of offsets and lead to lost opportunities (Trexler et al., 2006). This could potentially reduce much of the cost savings from including offsets in cap-and-trade programs. To determine the relationship among baseline stringencies, offsets supply and cost savings, we simulate the model under three baseline protocols. We define the protocol denoted 
by "Predicted BAU Emissions" by setting baselines equal to predicted BAU emissions. We call the second protocol "Minimize Supply of Over-Credited Offsets." This protocol sets baselines to ensure that there is no supply of over-credited offsets. The third protocol, "Maintain Environmental Integrity," adjusts baselines to the point where the aggregate supply of over-credited offsets equals the quantity of under-credited emissions reductions. Under this protocol, the effect of including offsets in the cap-and-trade program has no net effect on emissions as the two sources of emissions changes cancel.

Table 1 reports offsets supply and emissions consequences of including offsets in the cap-and-trade program for three capped sector reduction targets. Panels (a), (b) and (c) report estimates for a low, medium and high capped sector reduction target, respectively. In general, the higher the reduction target, the higher the equilibrium price of permits and offsets. This result is illustrated by comparing the equilibrium offset prices across the three panels. When the capped sector reduction target is low, equilibrium prices range from $\$ 7.66$ to $\$ 11.69$, while with a high capped sector reduction target, equilibrium prices range from $\$ 75.86$ to $\$ 85.85$.

Table 1 highlights three key findings. First, setting baselines equal to predicted BAU emissions leads to a substantial increase in emissions. For a low capped sector reduction target (Table 1, Panel (a)), there are only $4 \mathrm{MMTCO}_{2} \mathrm{e}$ under-credited emissions reductions, compared to $144 \mathrm{MMTCO}_{2} \mathrm{e}$ over-credited offsets, leading to an aggregate increase in emissions of $140 \mathrm{MMTCO}_{2} \mathrm{e}$. Emissions increase because projects with baselines above their BAU emissions opt in and receive over-credited offsets, while projects with baselines below their BAU emissions are not as likely to opt in and generate under-credited emissions reductions. Second, baseline protocols that attempt to fully eliminate the supply of over-credited offsets significantly reduce the supply of offsets. Across all three capped sector reduction target scenarios, we find that the minimize supply of over-credited offsets protocol has a much lower supply of offsets than the predicted BAU emissions protocol. For a capped sector reduction target of 2,000 $\mathrm{MMTCO}_{2} \mathrm{e}$, total offsets supply is about 50 percent less under the minimize supply of over-credited offsets protocol. Third, the maintain environmental integrity baseline protocol does not significantly reduce the supply of offsets as long as offset prices are high. For a capped sector reduction target of 3,500 $\mathrm{MMTCO}_{2} \mathrm{e}$, total offsets supply under the maintain environmental integrity protocol is $728 \mathrm{MMTCO}_{2} \mathrm{e}$, which is only 10 percent less than total offsets supply under the predicted BAU emissions protocol. High offset prices encourage a greater fraction of projects with baselines set below their BAU emissions. Greater participation by these projects increases the quantity of under-credited emissions reductions. As a consequence, as the equilibrium offsets price increases, there is less need for setting stringent baselines to balance the supply of over-credited offsets and the quantity of undercredited emissions reductions. This feature is illustrated by recognizing the required baseline stringencies for the different equilibrium offset prices. While low offset prices require very stringent baselines (Table 1, Panel 
(a), $b_{i}=0.46 \tilde{u}_{i}$ ), high offset prices provide room for leeway (Table 1, Panel (c), $b_{i}=0.77 \tilde{u}_{i}$ ). Moving from a low reduction target of $500 \mathrm{MMTCO}_{2} \mathrm{e}$ to a medium reduction target of 2,000 $\mathrm{MMTCO}_{2} \mathrm{e}$ allows the policy to relax baseline stringency by 50 percent. This suggests that for a one dollar increase in the equilibrium offsets price, baselines can be increased by between one and two percent to maintain the environmental integrity of the program.

Table 2 translates offsets supply and equilibrium prices from Table 1 into cost savings estimates from including offsets in the cap-and-trade program. We find that the protocol that minimizes the supply of over-credited offsets severely reduces the cost savings from incorporating offsets into the program. For a capped sector reduction target of 2,000 $\mathrm{MMTCO}_{2} \mathrm{e}$, cost savings are about 50 percent less relative to the predicted BAU emissions protocol (Table 2, Panel (b)). In contrast, the maintain environmental integrity protocol does not sacrifice much cost savings as long as the capped sector reduction target is sufficiently high. When the target is set to $3,500 \mathrm{MMTCO}_{2} \mathrm{e}$, cost savings are only about 10 percent less relative to the predicted BAU emissions protocol. This result stems from the fact that more stringent reduction targets generate a supply of offsets that are only slightly less under the maintain environmental integrity protocol (Table 1). The result suggests that the trade-off between environmental integrity and compliance cost savings is insignificant under aggressive emissions reduction targets.

\section{Sensitivity Analysis}

To understand how our results depend on key market characteristics, we perform sensitivity analysis by simulating market outcomes over a wide range of parameters. We vary the tightness of offsets project baselines, from 20 percent to 100 percent of predicted BAU emissions, and analyze the pattern of offsets supply and emissions changes stemming from the quantity of under-credited emissions reductions and the supply of over-credited offsets. Sensitivity analysis around the basic assumptions including BAU emissions uncertainty, the offsets mitigation supply curve, the correlation between key variables, systematic bias in predicting BAU emissions and different measures of transaction costs is reported in the Supplementary Material. In each section of sensitivity analysis, we report the ratio of under-credited emissions reductions to over-credited offsets, offset supplies for broad ranges of the parameters and how different offsets protocols affect the cost savings from including offsets in cap-and-trade programs. Supplementary Tables 7, 8 and 9 report key model outputs for scenarios when a larger supply of offsets is allowed into the program, which represents a setting with international offsets. In these simulations we assume that the supply of mitigation function is multiplied by a constant proportion. We consider a wide range of alternative scenarios, including 25 percent (expensive mitigation opportunities) and 400 percent (cheap mitigation opportunities). Values above 100 percent represent programs that incorporate international offsets. When there are cheaper mitigation opportunities from offsets projects, there will be a greater quantity of under-credited emissions 
reductions created (see Supplementary Table 8), implying that baselines can be made less stringent to ensure the environmental integrity of the program. We find that transaction costs play a minor role in determining the relative magnitudes of over-credited offsets and under-credited emissions reductions (see Sections 9.7 and 9.8 of the Supplementary Material).

\section{Conclusion}

Our results imply two key policy recommendations, both of which involve differentiating baseline stringency. First, as the problem of over-crediting becomes less severe over time as carbon prices are expected to increase, baseline-setting stringency can be relaxed to encourage a greater supply of offsets. Therefore short-run policies that impose conservative baseline-setting measures appear justified, while they may be less justified in the future. Second, our framework serves as a guide for differentiating baseline stringency across projects based on project characteristics. In our main analysis we have shown that project types that have lower predicted BAU emissions uncertainty require a less stringent baseline to maintain environmental integrity. In the Supplementary Material, we show that projects with lower marginal costs of mitigation, higher offsets supply potential or lower transaction costs require a less stringent baseline to maintain environmental integrity. In the Supplementary Material, we categorize popular project types along these dimensions.

In addition to the significant cost reductions that offsets bring, recent arguments for including them in cap-and-trade programs point to the importance of their co-benefits. For example, offsets may be worthwhile for their ability to encourage the development of adaptation and transition toward a low-carbon world (Dargusch and Thomas, 2012). Other experience with carbon offsetting suggests that programs can prevent biodiversity loss and serve as a payment for ecosystem services projects (Green and Minchin, 2012; Jack et al., 2007; Siikamaki et al., 2012). The additional non-GHG mitigation benefits may be valuable enough to warrant incorporating offsets in cap-and-trade programs even when over-credited offsets exceed undercredited emissions reductions. Baselines calculated here can be further relaxed to account for these additional co-benefits. 


\section{References}

Basu, P. (2009). Forestry: A green investment. Nature 457, 144-146.

Bento, A., R. Kanbur, and B. Leard (2015). Designing efficient markets for carbon offsets with distributional constraints. Journal of Environmental Economics and Management 70, 51-71.

Braun, N., T. Fitzgerald, and J. Pearcy (2015). Tradable emissions permits with offsets. SSRN Working Paper 2434984.

Brown, K. and W. Adger (1994). Economic and political feasibility of international carbon offsets. Forest Ecology Management 68(2-3), 217-229.

Busch, J., R. Lubowski, F. Godoy, M. Steininger, A. Yusuf, K. Austin, J. Hewson, D. Juhn, M. Farid, and F. Boltz (2012). Structuring economic incentives to reduce emissions from deforestation within Indonesia. Proceedings of the National Academy of Sciences 109(4), 1062-1067.

Bushnell, J. (2012). The economics of carbon offsets. National Bureau of Economic Research.

Calvin, K., S. Rose, M. Wise, H. McJeon, L. Clarke, and J. Edmonds (2015). Global climate, energy, and economic implications of international energy offsets. Climatic Change 133(4), 583-596.

Chameides, W. and M. Oppenheimer (2007). Carbon trading over taxes. Science 315, 1670.

Dargusch, P. and S. Thomas (2012). A critical role for carbon offsets. Nature Climate Change 2, 470.

EPA (2009a). Data annex coverage and caps: Emissions inventory - Scenario 7. http://www.epa.gov/ climatechange/economics/downloads/EPAactivities/HR2454Analysis-DataAnnex.zip.

EPA (2009b). EPA preliminary analysis of the Waxman-Markey discussion draft. http://www3.epa.gov/ climatechange/Downloads/EPAactivities/WM-Analysis.pdf.

EPA (2009c). Processed marginal abatement cost (MAC) curves.

EPA (2009d). Updated forestry and agriculture marginal abatement cost curves. http://www.epa.gov/ climatechange/economics/downloads/EPAactivities/HR2454Analysis-DataAnnex.zip.

Erickson, P., M. Lazarus, and R. Spalding-Fecher (2014). Net climate change mitigation of the clean development mechanism. Energy Policy 72, 146-154.

Fell, H., D. Burtraw, R. Morgenstern, and K. Palmer (2012). Soft and hard price collars in a cap-and-trade system: A comparative analysis. Journal of Environmental Economics and Management 64(2), 183-198.

Fell, H. and R. Morgenstern (2010). Alternative approaches to cost containment in a cap-and-trade system. Environmental and Resource Economics 47, 275-297.

Ferraro, P. (2008). Asymmetric information and contract design for payments for environmental services. Ecological Economics 65(4), 810-821.

Fischer, C. (2005). Project-based mechanisms for emissions reductions: Balancing trade-offs with baselines. Energy Policy 33, 1807-1823.

Gillenwater, M. (2012a). What is additionality? Part 1: A long standing problem. Greenhouse Gas Management Institute.

Gillenwater, M. (2012b). What is additionality? Part 2: A framework for a more precise definition and standardized approaches. Greenhouse Gas Management Institute.

Gillenwater, M., D. Broekhoff, M. Trexler, J. Hyman, and R. Fowler (2007). Policing the voluntary carbon market. Nature Reports: Climate Change 711(6), 85-87. 
Goulder, L., M. Hafstead, and M. Dworsky (2010). Impacts of alternative emissions allowance allocation methods under a federal cap-and-trade program. Journal of Environmental Economics and Management 60(3), 161-181.

Green, D. and L. Minchin (2012). The co-benefits of carbon management on country. Nature Climate Change: Commentary 2, 641-643.

Horowitz, J. and R. Just (2013). Economics of additionality for environmental services from agriculture. Journal of Environmental Economics and Management 66(1), 105-122.

Jack, B., C. Kousky, and K. Sims (2007). Designing payments for ecosystem services: Lessons from previous experience with incentive-based mechanisms. Proceedings of the National Academy of Sciences 105, 94459448.

Kile, J. (2009). The use of agricultural offsets to reduce greenhouse gases. Congressional Budget Office Testimony.

Kintisch, E. (2008). California emissions plan to explore use of offsets. Science 321, 23.

Lehmann, J. (2007). A handful of carbon. Nature 447, 143-144.

Mansanet-Bataller, M., J. Chevallier, M. Herve-Mignucci, and E. Alberola (2011). EUA and sCER phase II price drivers: Unveiling the reasons for the existence of the EUAsCER spread. Energy Policy 39(3), 1056-1069.

Maslin, M. (2011). Carbon trading needs a multi-level approach. Nature 475, 445-447.

Mason, C. and A. Plantinga (2013). The additionality problem with offsets: Optimal contracts for carbon sequestration in forests. Journal of Environmental Economics and Management 66, 1-14.

Menges, S. (2003). Supporting renewable energy on liberalised markets: Green electricity between additionality and consumer sovereignty. Energy Policy 75, 583-596.

Meyers, S. (1999). Additionality of emission reductions from clean development mechanism projects: Issues and options for project-level assessment. Lawrence Berkeley National Laboratory.

Millard-Ball, A. (2013). The trouble with voluntary emissions trading: Uncertainty and adverse selection in sectoral crediting programs. Journal of Environmental Economics and Management 65 (1), 40-55.

Montero, J.-P. (1999). Voluntary compliance with market-based environmental policy: Evidence from the U.S. acid rain program. Journal of Political Economy 107(5), 998-1033.

Montero, J.-P. (2000). Optimal design of a phase-in emissions trading program. Journal of Public Economics 75(2), 273-291.

Naegele, H. (2015). Offset credits in the EU ETS: A quantile estimation of firm-level transaction costs. DIW Discussion Paper.

Rentz, H. (1998). Joint implementation and the question of "additionality" - a proposal for a pragmatic approach to identify possible joint implementation projects. Energy Policy 26, 275-279.

Rubin, J. (1996). A model of intertemporal emission trading, banking and borrowing. Journal of Environmental Economics and Management 31, 269-286.

Schneider, L. (2009a). Assessing the additionality of CDM projects: Practical experiences and lessons learned. Climate Policy 9(3).

Schneider, L. (2009b). A Clean Development Mechanism with global atmospheric benefits for a post-2012 climate regime. International Environmental Agreements: Politics, Law and Economics 9, 95-111.

Siikamaki, J., J. Sanchirico, and S. Jardine (2012). Global economic potential for reducing carbon dioxide emissions from mangrove loss. Proceedings of the National Academy of Sciences 109, 14369-14374. 
Trexler, M., D. Broekhoff, and L. Kosloff (2006). A statistically-driven approach to offset-based GHG additionality determinations. What can we learn? Sustainable Development Law Policy 6, 31-40.

Van Benthem, A. and S. Kerr (2013). Scale and transfers in international emissions offset programs. Journal of Public Economics 107, 31-46.

Victor, D. (2012). National effects of a global policy. Nature Climate Change 2, 24-25.

Warnecke, C., S. Wartmann, N. Hohne, and K. Blok (2014). Beyond pure offsetting: Assessing options to generate net-mitigation-effects in carbon market mechanisms. Energy Policy 68, 413-422.

Zhang, J. and C. Wang (2011). Co-benefits and additionality of the clean development mechanism: An empirical analysis. Journal of Environmental Economics and Management 62(2), 140-154. 
Table 1 | The effect of alternative baseline protocols on offsets supply and emissions.

\begin{tabular}{|c|c|c|c|}
\hline $\begin{array}{l}\text { (a) Capped Sector Reduction Target } \\
=500 \mathrm{MMTCO}_{2} \mathrm{e}\end{array}$ & $\begin{array}{c}\text { Predicted } \\
\text { BAU Emissions }\end{array}$ & $\begin{array}{l}\text { Minimize Supply of } \\
\text { Over-Credited Offsets }\end{array}$ & $\begin{array}{l}\text { Maintain Environmental } \\
\text { Integrity }\end{array}$ \\
\hline Baselines & $b_{i}=\tilde{u}_{i}$ & $b_{i}=0$ & $b_{i}=0.46 \tilde{u}_{i}$ \\
\hline Offsets Price & 7.66 & 11.69 & 10.57 \\
\hline Percentage of Projects Opting In & 51 & 7 & 23 \\
\hline Total Offsets Supply & 202 & 86 & 127 \\
\hline Exact Offsets & 58 & 86 & 109 \\
\hline Over-Credited Offsets & 144 & 0 & 17 \\
\hline Under-Credited Emissions Reductions & 4 & 27 & 17 \\
\hline Total Change in Emissions & 140 & -27 & 0 \\
\hline $\begin{array}{r}\text { (b) Capped Sector Reduction Target } \\
=2,000 \mathrm{MMTCO}_{2} \mathrm{e}\end{array}$ & $\begin{array}{c}\text { Predicted } \\
\text { BAU Emissions }\end{array}$ & $\begin{array}{l}\text { Minimize Supply of } \\
\text { Over-Credited Offsets }\end{array}$ & $\begin{array}{l}\text { Maintain Environmental } \\
\text { Integrity } \\
\end{array}$ \\
\hline Baselines & $b_{i}=\tilde{u}_{i}$ & $b_{i}=0$ & $b_{i}=0.70 \tilde{u}_{i}$ \\
\hline Offsets Price & 38.14 & 47.02 & 40.83 \\
\hline Percentage of Projects Opting In & 66 & 30 & 57 \\
\hline Total Offsets Supply & 652 & 338 & 556 \\
\hline Exact Offsets & 505 & 338 & 497 \\
\hline Over-Credited Offsets & 147 & 0 & 59 \\
\hline Under-Credited Emissions Reductions & 40 & 112 & 59 \\
\hline Total Change in Emissions & 107 & -112 & 0 \\
\hline $\begin{array}{r}\text { (c) Capped Sector Reduction Target } \\
=3,500 \mathrm{MMTCO}_{2} \mathrm{e}\end{array}$ & $\begin{array}{c}\text { Predicted } \\
\text { BAU Emissions }\end{array}$ & $\begin{array}{l}\text { Minimize Supply of } \\
\text { Over-Credited Offsets }\end{array}$ & $\begin{array}{l}\text { Maintain Environmental } \\
\text { Integrity }\end{array}$ \\
\hline Baselines & $b_{i}=\tilde{u}_{i}$ & $b_{i}=0$ & $b_{i}=0.77 \tilde{u}_{i}$ \\
\hline Offsets Price & 75.86 & 85.85 & 78.43 \\
\hline Percentage of Projects Opting In & 74 & 39 & 68 \\
\hline Total Offsets Supply & 817 & 436 & 728 \\
\hline Exact Offsets & 672 & 436 & 653 \\
\hline Over-Credited Offsets & 147 & 0 & 75 \\
\hline Under-Credited Emissions Reductions & 61 & 152 & 75 \\
\hline Total Change in Emissions & 86 & -152 & 0 \\
\hline
\end{tabular}

Carbon offset prices are reported in dollars per ton of $\mathrm{CO}_{2} \mathrm{e}$. Offsets supply, emissions reductions and changes in emissions are reported in $\mathrm{MMTCO}_{2} \mathrm{e}$. The percentage of projects opting in is the ratio of the quantity of projects that are opted in to the quantity of all potential projects. The Predicted BAU Emissions protocol is defined by setting project baselines equal to predicted BAU emissions. The Minimize Supply of Over-Credited Offsets protocol is defined by setting project baselines that guarantee zero supply of over-credited offsets. The Maintain Environmental Integrity protocol is defined by setting project baselines such that the expected supply of over-credited offsets equals the expected quantity of undercredited emissions reductions. This protocol keeps expected aggregate emissions fixed. Each panel shows average outcomes from 2,000 simulations. In each simulation the offsets price is endogenously determined by equating the supply and demand for offsets (see Supplementary Material). 
Table $2 \mid$ The cost savings from including offsets in cap-and-trade programs under alternative baseline protocols.

\begin{tabular}{|c|c|c|c|c|}
\hline $\begin{array}{r}\text { (a) Capped Sector Reduction Target } \\
=500 \mathrm{MMTCO}_{2} \mathrm{e}\end{array}$ & No Offsets & $\begin{array}{c}\text { Predicted } \\
\text { BAU Emissions }\end{array}$ & $\begin{array}{c}\text { Minimize Supply of } \\
\text { Over-Credited Offsets }\end{array}$ & $\begin{array}{l}\text { Maintain Environmental } \\
\text { Integrity }\end{array}$ \\
\hline Capped Sector Mitigation & 500 & 272 & 413 & 373 \\
\hline Offsets Supply & 0 & 204 & 86 & 127 \\
\hline Capped Sector Mitigation Costs & 3,538 & 1,048 & 2,420 & 1,969 \\
\hline Uncapped Sector Mitigation Costs & 0 & 101 & 314 & 340 \\
\hline Uncapped Sector Transaction Costs & 0 & 1,019 & 430 & 636 \\
\hline Total Compliance Costs & 3,538 & 2,169 & 3,164 & 2,946 \\
\hline Cost Savings & - & 1,369 & 374 & 592 \\
\hline $\begin{array}{r}\text { (b) Capped Sector Reduction Target } \\
=2,000 \mathrm{MMTCO}_{2} \mathrm{e}\end{array}$ & "No Offsets & $\begin{array}{c}\text { Predicted } \\
\text { BAU Emissions }\end{array}$ & $\begin{array}{c}\text { Minimize Supply of } \\
\text { Over-Credited Offsets }\end{array}$ & $\begin{array}{l}\text { Maintain Environmental } \\
\text { Integrity }\end{array}$ \\
\hline Capped Sector Mitigation & 2,000 & 1,350 & 1,661 & 1,442 \\
\hline Offsets Supply & 0 & 650 & 339 & 557 \\
\hline Capped Sector Mitigation Costs & 56,600 & 25,784 & 39,020 & 29,419 \\
\hline Uncapped Sector Mitigation Costs & 0 & 7,172 & 6,034 & 7,524 \\
\hline Uncapped Sector Transaction Costs & 0 & 3,249 & 1,697 & 2,785 \\
\hline Total Compliance Costs & 56,600 & 36,206 & 46,751 & 39,728 \\
\hline Cost Savings & - & 20,394 & 9,849 & 16,872 \\
\hline $\begin{array}{r}\text { (c) Capped Sector Reduction Target } \\
=3,500 \mathrm{MMTCO}_{2} \mathrm{e}\end{array}$ & No Offsets & $\begin{array}{c}\text { Predicted } \\
\text { BAU Emissions }\end{array}$ & $\begin{array}{c}\text { Minimize Supply of } \\
\text { Over-Credited Offsets }\end{array}$ & $\begin{array}{l}\text { Maintain Environmental } \\
\text { Integrity }\end{array}$ \\
\hline Capped Sector Mitigation & 3,500 & 2,678 & 3,031 & 2,769 \\
\hline Offsets Supply & 0 & 817 & 434 & 730 \\
\hline Capped Sector Mitigation Costs & 173,338 & 101,488 & 130,022 & 108,499 \\
\hline Uncapped Sector Mitigation Costs & 0 & 15,036 & 11,507 & 15,115 \\
\hline Uncapped Sector Transaction Costs & 0 & 4,084 & 2,168 & 3,649 \\
\hline Total Compliance Costs & 173,338 & 120,608 & 143,698 & 127,263 \\
\hline Cost Savings & - & 52,730 & 29,640 & 46,075 \\
\hline
\end{tabular}

Capped and uncapped sector mitigation are reported in $\mathrm{MMTCO}_{2} \mathrm{e}$. Costs and cost savings estimates are reported in millions of (year 2000) dollars. The Predicted BAU Emissions protocol is defined by setting project baselines equal to predicted BAU emissions. The Minimize Supply of Over-Credited Offsets protocol is defined by setting project baselines that guarantee zero supply of over-credited offsets. The Maintain Environmental Integrity protocol is defined by setting project baselines such that the expected supply of over-credited offsets equals the expected quantity of under-credited emissions reductions. This protocol keeps expected aggregate emissions fixed. Each panel shows average outcomes from 2,000 simulations. In each simulation the offsets price is endogenously determined by equating the supply and demand for offsets (see Supplementary Material). 




Figure 1 Emissions and offsets supply consequences from the decisions of uncapped sector project managers. The horizontal axis denotes the ratio of a project's assigned baseline $\left(b_{i}\right)$ and its BAU emissions $\left(u_{i}\right)$. The vertical axis measures a project's marginal cost of mitigation $\left(c_{i}\right)$, where the horizontal line $p-t_{i}$ represents the equilibrium price of offsets minus the project's transaction cost per ton of $\mathrm{CO}_{2} \mathrm{e}$. The equilibrium price of offsets is determined as an endogenous variable in our simulation model. Managers of projects that are classified in the blue and purple regions do not opt in their projects. The green region includes projects that produce under-credited emissions reductions. Projects belonging to the red and orange regions supply over-credited offsets. The curve separating the blue and green regions represents a zero-profit condition of the project profit-maximization problem (see the Supplementary Material for a formal definition and derivation). 


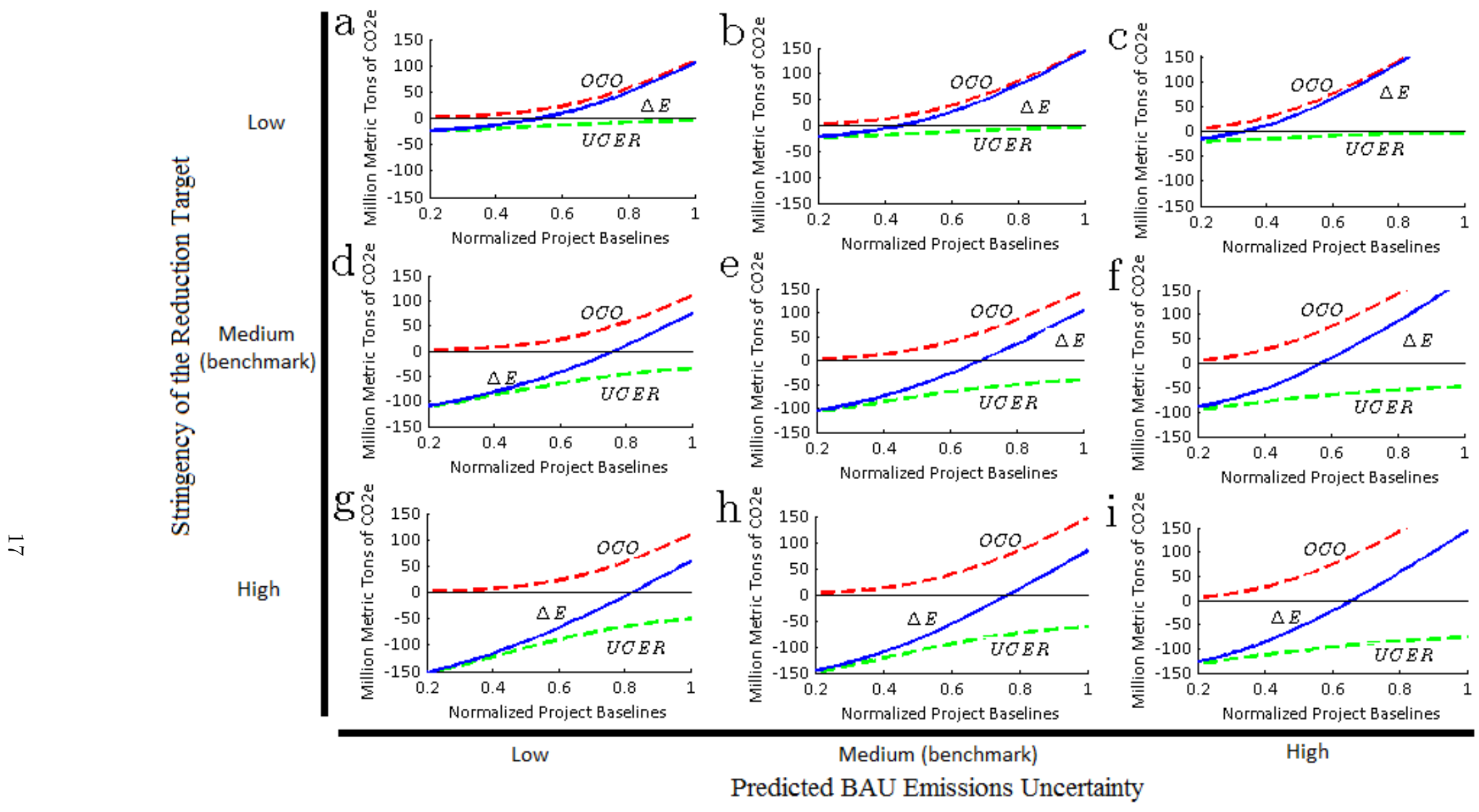

Figure 2 $\mid$ The change in aggregate emissions relative to a program that does not include offsets, as a function of normalized project baselines. In each panel, the horizontal axis measures normalized project baselines, which are defined as a project's assigned baseline $\left(b_{i}\right)$ divided by the project's predicted BAU emissions $\left(\tilde{u}_{i}\right)$. The vertical axis measures million metric tons of $\mathrm{CO}_{2} \mathrm{e}$. The change in emissions $(\Delta E)$ is defined relative to a cap-and-trade program that does not include offsets. Its value is calculated by adding the supply of over-credited offsets $(O C O)$ and the quantity of under-credited emissions reductions $(U C E R)$. Panels in the same row are simulations of programs that have a common capped sector reduction target. We consider three targets: low (500 $\left.\mathrm{MMTCO}_{2} \mathrm{e}, \mathbf{a}, \mathbf{b}, \mathbf{c}\right)$, medium $\left(2,000 \mathrm{MMTCO}_{2} \mathrm{e}, \mathbf{d}, \mathbf{e}, \mathbf{f}\right)$ and high $\left(3,500 \mathrm{MMTCO}_{2} \mathrm{e}\right.$, $\mathbf{g}, \mathbf{h}, \mathbf{i})$. Panels in the same column are simulations that have the same uncertainty on predicted BAU emissions. We consider three levels of predicted BAU uncertainty that are defined by the standard deviation of emissions shocks $(\sigma)$. Our benchmark simulation assumes that the standard deviation of prediction errors for BAU emissions is equal to the expected value of BAU emissions $(\sigma=E[u], \mathbf{b}, \mathbf{e}, \mathbf{h})$. The remaining cases have a low level of uncertainty $(\sigma=0.75 E[u]$, a,d,g $)$ and high level of uncertainty $(\sigma=1.5 E[u], \mathbf{c}, \mathbf{f}, \mathbf{i})$. Each panel shows average outcomes from 2,000 simulations. In each simulation the offsets price is endogenously determined by equating the supply and demand for offsets (see Supplementary Material). 


\section{Supplementary Material for On the importance of baseline setting in carbon offsets markets}




\section{Contents}

1 Introduction $\quad 4$

2 Analytical Framework $\quad 5$

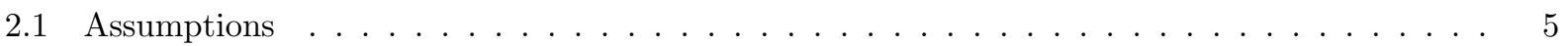

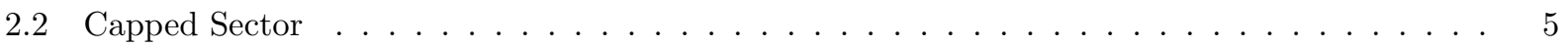

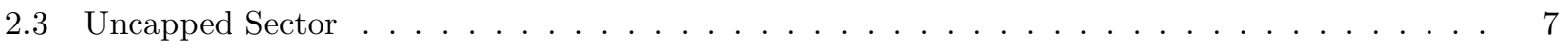

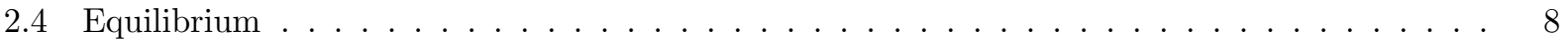

3 Emissions Effects $\quad 10$

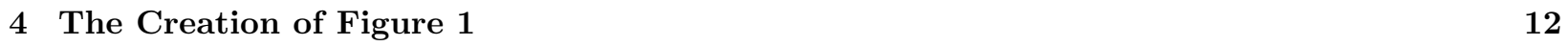

5 The Effect of Project Characteristics and Market Conditions on Potential Project $\begin{array}{ll}\text { Decisions } & 14\end{array}$

5.1 Offsets Supply Potential . . . . . . . . . . . . . . . . . . . . . 14

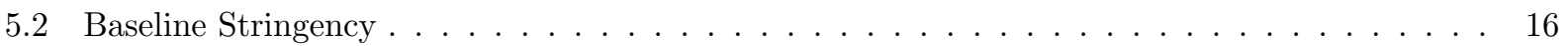

$5.3 \quad$ Net Carbon Price . . . . . . . . . . . . . . . . . . . . . . 18

6 Calibration $\quad 20$

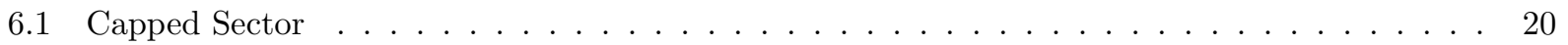

6.2 Uncapped Sector . . . . . . . . . . . . . . . . . . . . . . . . . . 21

7 Numerical Model Equilibrium and Output $\quad 27$

8 Model Validation $\quad 28$

9 Sensitivity Analysis $\quad 31$

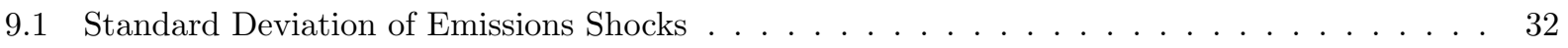

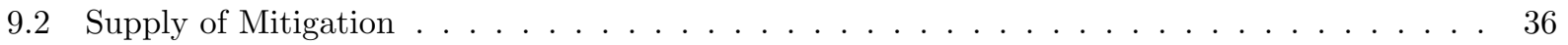

9.3 Correlation Between BAU Emissions and Sequestration Potential . . . . . . . . . . . . . 41

9.4 Correlation Between BAU Emissions and Marginal Costs of Mitigation . . . . . . . . . . . 45

9.5 Correlation Between Sequestration Potential and Marginal Costs of Mitigation . . . . . . 50

9.6 Systematic Bias in Predicted BAU Emissions $\ldots \ldots \ldots \ldots \ldots$

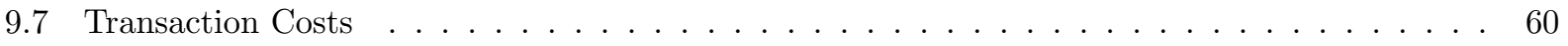

9.8 Transaction Costs as a Fraction of Total Implementation Costs . . . . . . . . . . . . . . 65 


\section{Introduction}

The supporting information includes a detailed description of our analytical framework that underpins the numerical model; definitions of the emissions effects; a formal derivation of the equations that are illustrated in Figure 1; a comprehensive description of how we calibrate the analytical model with values from the literature; a description of the numerical model equilibrium and output; model validation that compares benchmark simulation output to other studies in the literature; a section on sensitivity analysis; and a section describing how our sensitivity analysis links back to carbon offsets project types. 


\section{Analytical Framework}

Here we develop an analytical model to establish the behavior of the economic agents in the model and to define how we calculate the supply of offsets, the supply of over-credited offsets, the quantity of under-credited reductions, equilibrium prices of offsets and permits, and cost savings from including offsets in cap-and-trade programs.

\subsection{Assumptions}

Our simulation results are based on an analytical model that links a capped sector with an uncapped sector through a market for carbon offsets. The uncapped sector is composed of heterogeneous projects. Managers of these projects make profit-maximizing decisions to have their projects opt in to the program by supplying offsets to the capped sector. The capped sector is represented by a single cost-minimizing firm. The sector complies with a cap-and-trade program by abating its emissions and purchasing offsets from the uncapped sector.

\subsection{Capped Sector}

The capped sector represents industries likely to be covered under a federal greenhouse gas (GHG) cap-and-trade program. We base our representation on the industries that would have been covered under the H.R. 2454, the American Clean Energy and Security Act, henceforth the Waxman-Markey bill, which include coal-fired power plants, petroleum refineries, natural gas refineries, iron and steel production and cement manufacture. The capped sector is regulated by a cap-and-trade program. We model the capped sector as a representative firm that takes equilibrium prices as given. This is a standard assumption used to evaluate compliance costs of cap-and-trade programs (Fell and Morgenstern, 2010; Fell et al., 2012). In addition, this approach mimics the outcome of a set of competitive firms (Lucas and Prescott, 1971; Baldursson and Karatzas, 1997). The capped sector is allocated a fixed quantity of emissions permits that is equal to capped sector business-as-usual (BAU) 
emissions minus a reduction target denoted by $\bar{q}$. To comply with the cap, the capped sector solves the following constrained cost minimization problem:

$$
\begin{aligned}
& T C=\min _{q, f}\{T A C(q)+p f\} \quad \text { subject to } \quad \text { (Supplementary Equation 1) } \\
& q+f \geq \bar{q} .
\end{aligned}
$$

The objective of the capped sector is to minimize total compliance costs $(T C)$, which equal the sum of total abatement costs $T A C(\cdot)$ and the cost of purchasing offsets. The cost of purchasing offsets is the product of the number of offsets purchased $(f)$ and the equilibrium offsets price $(p)$. The capped sector chooses how much to abate $(q)$ and how many offsets to buy to minimize total compliance costs subject to meeting the reduction target, $q+f \geq \bar{q}$. Note that we do not explicitly model the equilibrium permit price. This is because we represent the capped sector as a single, perfectly competitive firm. The equilibrium outcome from our model is identical to the equilibrium outcome from a set of perfectly competitive firms that can trade permits (Fell and Morgenstern, 2010; Fell et al., 2012). If we were to explicitly provide a permit price, it would be equal to the marginal cost of abatement of the capped sector in equilibrium (Baumol and Oates, 1988). The two conditions for an optimal solution imply that

$$
T A C^{\prime}\left(q^{*}\right)=p,
$$

(Supplementary Equation 3)

or that the capped sector optimal abatement $q^{*}$ is where the marginal cost of abatement, $T A C^{\prime}(\cdot)$, equals the equilibrium offsets price. Therefore in equilibrium, the permit and offset prices are identical. Distortions that we do not consider in our model, such as a trade ratio or a limit on the use of offsets, would put a wedge between these prices.

We assume that $T A C^{\prime}(\cdot)$ is invertible so that condition in Supplementary Equation 3 can be solved for $q^{*}$ as a function of $p: q^{*}=q^{*}(p)$. This function defines the equilibrium quantity of abatement by the capped sector. 


\subsection{Uncapped Sector}

We assume that there are $n$ potential projects indexed by $i=1,2, \ldots, n$. Each project is managed independently. In our model, managers are the decision makers and are indexed by which project they control, $i=1,2, \ldots, n$. A manager will decide what to do with its potential project based on four project-specific characteristics and the equilibrium offsets price. The four characteristics include the marginal costs of mitigation $\left(c_{i}\right)$, BAU emissions $\left(u_{i}\right)$, sequestration potential $\left(s_{i}\right)$ and an emissions baseline $\left(b_{i}\right)$. Marginal costs are constant

and are drawn from a cumulative distributional function $Z(c)$ with support $[\underline{c}, \bar{c}]$. BAU emissions lie within a support $[\underline{u}, \bar{u}]$ where each $u_{i}$ is independently drawn from the cumulative distribution function $Y(u)$. Project $i$ 's sequestration potential is drawn from a cumulative distribution function $X(s)$ that has a support $[\underline{s}, \bar{s}]$, where $\underline{s}<0$ and $\bar{s}<0$.

Manager $i$ observes its project's marginal cost of mitigation, BAU emissions and sequestration potential. We assume that the policy maker measures BAU emissions $\tilde{u}_{i}$ of each project with uncertainty. Project $i$ 's predicted BAU emissions, denoted by $\tilde{u}_{i}$, are equal to BAU emissions plus an emissions shock $\varepsilon_{i} \sim \mathcal{N}\left(0, \sigma^{2}\right)$ :

$$
\tilde{u}_{i}=u_{i}+\varepsilon_{i} .
$$

(Supplementary Equation 4)

Each project receives a baseline, $b_{i}$, that equals a proportion of predicted BAU emissions:

$$
b_{i}=\alpha \tilde{u}_{i}
$$

The proportion $\alpha$ can be less than, equal to or greater than one. Managers make opt-in and mitigation decisions for their projects based on the profit function

$$
\pi_{i}=\max _{s_{i} \leq e_{i} \leq u_{i}}\left\{\left(p-t_{i}\right)\left(b_{i}-e_{i}\right)-c_{i}\left(u_{i}-e_{i}\right)\right\}, \quad \text { (Supplementary Equation 6) }
$$

where $p$ is the price of offsets and $t_{i}$ is a project-specific transaction cost per offset awarded. If $\pi_{i} \geq 0$, then manager $i$ opts in its project to supply a quantity of offsets equal to 
$f_{i}^{*}=b_{i}-e_{i}^{*}$, where $e_{i}^{*}$ solves Supplementary Equation 6 . Note that $e_{i}^{*}$ can be positive or negative, depending on the profitability of each action. A project that sequesters emissions has $e_{i}^{*}=s_{i}<0$. A project that has $e_{i}$ set to BAU emissions has $e_{i}^{*}=u_{i}>0$. This implies that even if $b_{i}=0$, project $i$ can supply a positive quantity of offsets. In this case, potential offsets supply from project $i$ is equal to the absolute value of $s_{i}$. Finally we assume that the policy maker perfectly measures ex-post emissions $e_{i}^{*}$ for each project $i$.

The supply of offsets from project $i$, denoted by $f_{i}^{s}$, is given by

$$
f_{i}^{s}= \begin{cases}b_{i}-e_{i}^{*}, & \text { if } b_{i}-e_{i}^{*}>0 \\ 0 & \text { otherwise }\end{cases}
$$

Since each project has a negative sequestration potential $s_{i}<0$, even if the project's assigned baseline is equal to zero, it can still mitigate emissions through sequestration, $e_{i}^{*}=s_{i}<0$. The total supply of offsets, denoted by $f$, is defined as the sum of offsets from each project:

$$
f^{s}=\sum_{i=1}^{n} f_{i}^{s}\left(p, b_{i}, s_{i}, u_{i}, t_{i}\right) .
$$

Since the decision of each manager is dependent on the equilibrium price of offsets, we denote the supply of offsets as a function of this price: $f^{s}=f^{s}(p)$.

\subsection{Equilibrium}

We define an equilibrium as an offsets price that equates the demand for offsets and the supply of offsets:

$$
f^{*}=f^{s}(p) .
$$

(Supplementary Equation 9)

Plugging this condition into the constraint Supplementary Equation 2 and recognizing that the constraint will be binding at a capped sector problem optimal solution $\left(q^{*}, f^{*}\right)$, we have

$$
q^{*}(p)+f^{s}(p)=\bar{q} .
$$


In our simulation model we assign functional forms to the model's equations so that the functions $q^{*}(\cdot)$ and $f^{s}(\cdot)$ satisfy sufficient conditions for a unique $p$ to satisfy Supplementary Equation 10. This price will define the equilibrium of our model. Given the equilibrium price, we can calculate capped firm abatement, the supply of offsets and emissions effects, which we define in the next section. 


\section{$3 \quad$ Emissions Effects}

With this framework, we derive the impact of allowing the capped sector to use offsets for compliance on emissions. We define the impact relative to a hypothetical program that does not permit the capped sector to use offsets. If offsets are allowed to be used for compliance, aggregate emissions may increase or decrease relative to this hypothetical. The change in emissions is dependent on the relative magnitudes of over-credited offsets and under-credited emissions reductions. Next we define these concepts in the context of our model. First we distinguish between two types of offsets: exact and over-credited. Exact offsets are offsets that correspond to emissions reductions. The supply of exact offsets from project $i$, denoted by $f_{i}^{E}$, is given by the difference between project $i$ 's BAU emissions and its emissions choice:

$$
f_{i}^{E}=u_{i}-e_{i}^{*}
$$

The total supply of exact offsets, denoted by $f^{E}$, is defined as the sum of exact offsets from each project:

$$
f^{E}=\sum_{i=1}^{n} f_{i}^{E} .
$$

Over-credited offsets are offsets that do not correspond to emissions reductions. The supply of over-credited offsets from project $i$, denoted by $f_{i}^{O C}$, is given by

$$
f_{i}^{O C}= \begin{cases}b_{i}-u_{i}, & \text { if } b_{i}-u_{i}>0 \\ 0 & \text { otherwise }\end{cases}
$$

The total supply of over-credited offsets, denoted by $F^{O C}$, is defined as the sum of overcredited offsets from each project:

$$
F^{O C}=\sum_{i=1}^{n} f_{i}^{O C}
$$


The quantity of under-credited emissions reductions from project $i$, denoted by $r_{i}$, is given by

$$
r_{i}= \begin{cases}b_{i}-u_{i}, & \text { if } b_{i}-u_{i}<0 \text { and } e_{i}^{*}<u_{i}^{*} \\ 0 & \text { otherwise }\end{cases}
$$

The total quantity of under-credited emissions reductions, denoted by $R$, is defined as the sum of under-credited emissions reductions from each project:

$$
R=\sum_{i=1}^{n} r_{i}
$$

The change in emissions relative to a program without offsets, $\Delta E$, equals the total supply of over-credited offsets plus the total quantity of under-credited emissions reductions:

$$
\Delta E=F^{O C}+R .
$$

(Supplementary Equation 17) 


\section{The Creation of Figure 1}

Figure 1 is constructed by solving the problem of manager $i$ in Supplementary Equation 6 . If $c_{i}>p-t_{i}$, then project $i$ 's marginal cost of mitigation exceeds the net marginal return of mitigation. Therefore the manager has its project perform no mitigation by selecting $e_{i}=u_{i}$. In this case, profits are

$$
\pi_{i}=\left(p-t_{i}\right)\left(b_{i}-u_{i}\right)
$$

If $b_{i}<u_{i}$, indicated by the purple and blue cross-hashed regions in Figure 1, then $\pi_{i}<0$. In this case, the manager of project $i$ will not opt in its project and will not have it perform mitigation. If $b_{i}>u_{i}$, indicated by the red region in Figure 1 , then $\pi_{i}>0$. In this case, manager $i$ will opt in its project but will not have it perform mitigation.

Now consider a project that has $c_{i}<p-t_{i}$. For this project, the marginal cost of mitigation is less than the marginal return of mitigation for project $i$. If $b_{i}>u_{i}$, indicated by the orange region in Figure 1, then $\pi_{i}>0$. In this case, manager $i$ will opt in its project and will have it mitigate by selecting $e_{i}=s_{i}$. If $b_{i}<u_{i}$, represented by the blue and green regions, then the manager's decision depends on the sign of Supplementary Equation 6. The manager will opt in its project and have it mitigate emissions if the returns exceed the costs. The necessary condition for manager $i$ to opt in its project is

$$
\left(p-t_{i}\right)\left(b_{i}-s_{i}\right)-c_{i}\left(u_{i}-s_{i}\right) \geq 0 \text {. }
$$

The left-hand side represents project $i$ 's profit if its manager chooses $e_{i}=s_{i}$, while the right-hand side represents project $i$ 's profit if the manager does not opt it in. Solving Supplementary Equation 19 for $c_{i}$ yields

$$
c_{i} \leq \frac{\left(p-t_{i}\right)\left(b_{i}-s_{i}\right)}{u_{i}-s_{i}}
$$

The non-linear curve in Figure 1 represents the case when Supplementary Equation 20 is 
binding. Managers of projects with marginal costs above the curve do not find it profitable to opt in their projects and mitigate emissions, represented by the blue region, while those managers of projects with marginal costs below the curve achieve positive net revenue from opting in and mitigating emissions, represented by the green region. 


\section{The Effect of Project Characteristics and Market Conditions on Potential Project Decisions}

To better understand how project characteristics and market conditions influence the decisions of project managers, we present alternatives of Figure 1 under different scenarios. First, we adjust offsets supply potential of a potential project. Second, we adjust baseline stringencies. Third, we present Figure 1 under several different market conditions, where projects face different carbon prices or transaction costs.

\subsection{Offsets Supply Potential}

Supplementary Figure 1 displays versions of Figure 1 that illustrate different offsets supply potentials. In Supplementary Figure 1, we vary sequestration potential $s_{i}$ of potential project $i$, which maps directly into offsets supply potential: a one unit increase in sequestration potential increases offsets supply potential by one unit. Moving from Panels (a) to (c) represents an increase in supply potential, where a hypothetical project described in Panel (a) has a low supply potential. Projects with low supply potential are less likely to opt in (illustrated by the relatively large blue region) and are less likely to be under-credited (illustrated by the relatively small green region). The remaining regions are unaffected. This finding suggests that project types that have a relatively large supply potential are likely to create more under-credited emissions reductions. 


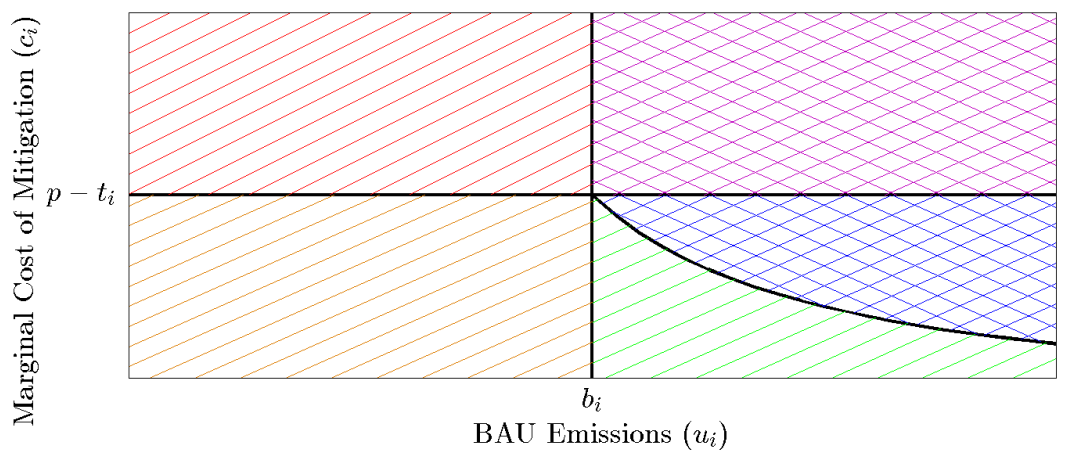

(a) Low Offsets Supply Potential

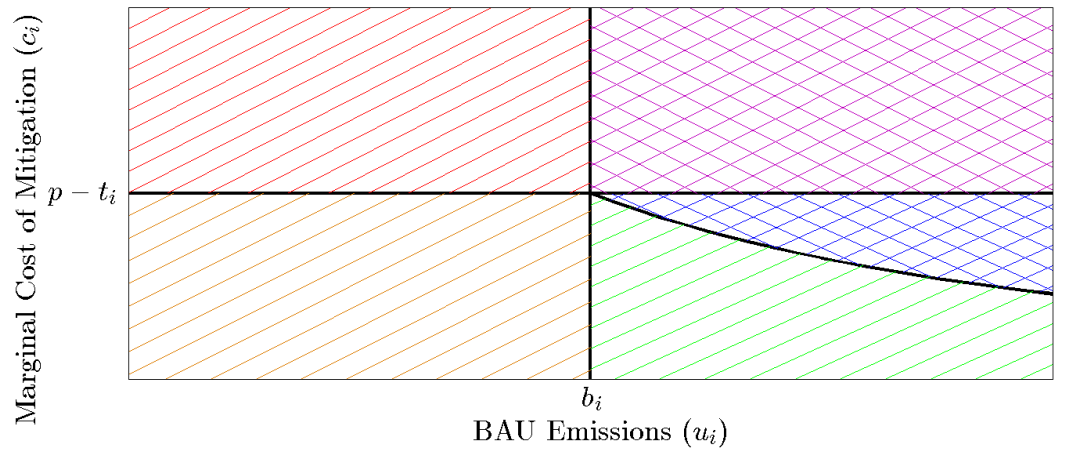

(b) Medium Offsets Supply Potential

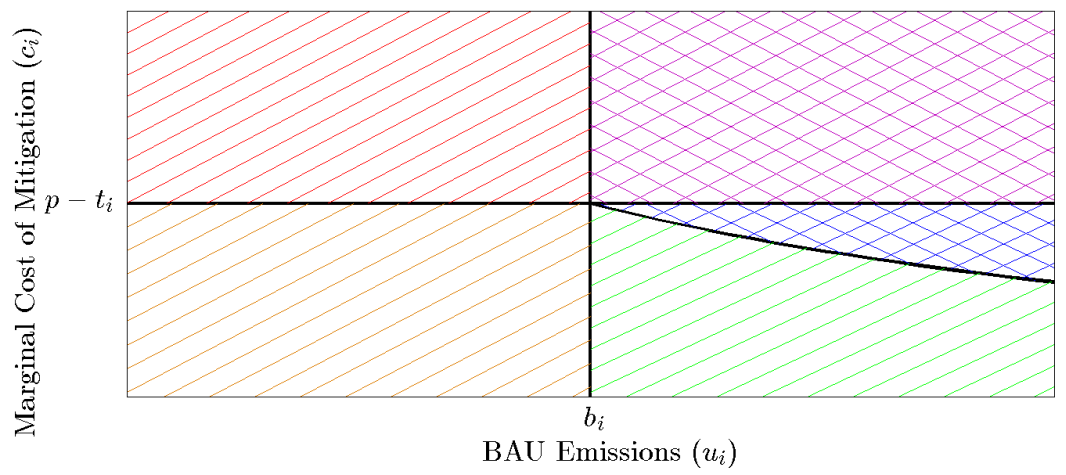

(c) High Offsets Supply Potential

Supplementary Figure $1 \mid$ The effect of offsets supply potential on potential project decisions. The Low Offsets Supply Potential case (a) is defined by a potential project having a relatively small (in absolute value) sequestration potential, $s_{i}$. The High Offsets Supply Potential case (c) is defined by a potential project having a relatively large (in absolute value) sequestration potential. 


\subsection{Baseline Stringency}

Supplementary Figure 2 displays versions of Figure 1 that illustrate different baseline stringencies. In Supplementary Figure 1, we vary the baseline assigned to project $i, b_{i}$, from a high (lenient) baseline in Panel (a) to a more conservative, low baseline in Panel (c). Several areas are influenced by the baseline choice. First, as baselines become more stringent (moving from (a) to (c)), the red and orange areas representing projects that are over-credited shrink. This is because projects that face a relatively low baseline are less likely to have BAU emissions that lie below its baseline. Second, as baselines become more stringent, the purple and blue areas representing projects that are not opted in grow. This is because potential projects that face a relatively low baseline has less of a profit incentive to be opted in. Third, with a more stringent baseline, the green area representing projects that opt in and that are under-credited may shrink or grow. This is due to two effects. The first effect is that a lower baseline makes it more likely that a potential project will be under-credited if it is opted in. This stretches the green area horizontally as seen by moving from Panel (a) to Panel (c). The second countervailing effect is that a lower baseline reduces the incentive for a project with BAU emissions above its assigned baseline to be opted in, which has the effect of reducing the relative green area and increasing the relative blue area in the lower-right quadrant of Figure 1. In essence, a lower baseline makes it more likely that a project opting in is under-credited, but it also discourages projects from being opted in.

Given that more conservative baselines reduce the over-credited areas and has an ambiguous effect on the under-credited area, we recognize that a marginal reduction of project baselines will likely increase the ratio of under-credited emissions reductions to overcredited offsets. If, however, baselines are reduced enough, the entire area of Figure 1 will become dominated by the blue and purple regions as virtually no projects are opted in. Therefore this conclusion is limited to the extent that some projects are still worth opting in. 


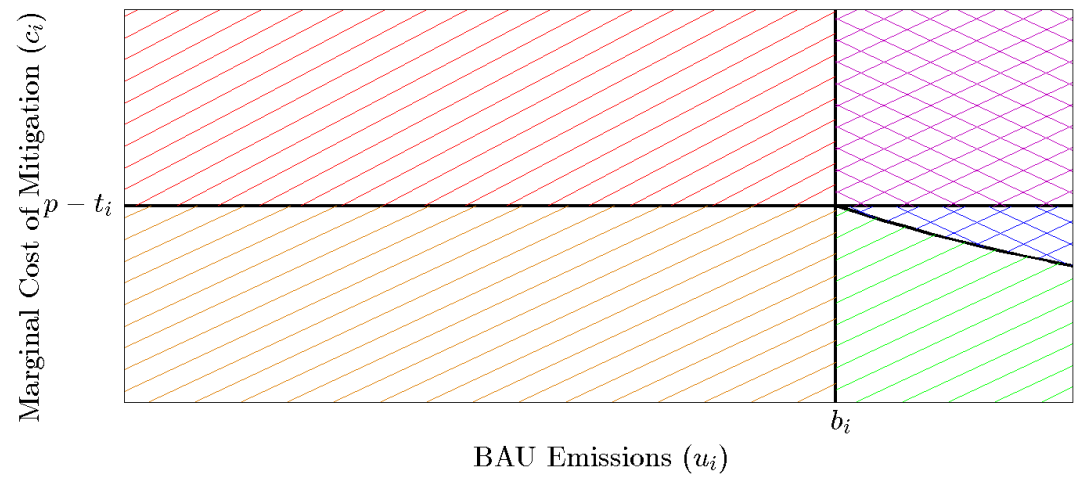

(a) High (Lenient) Baseline

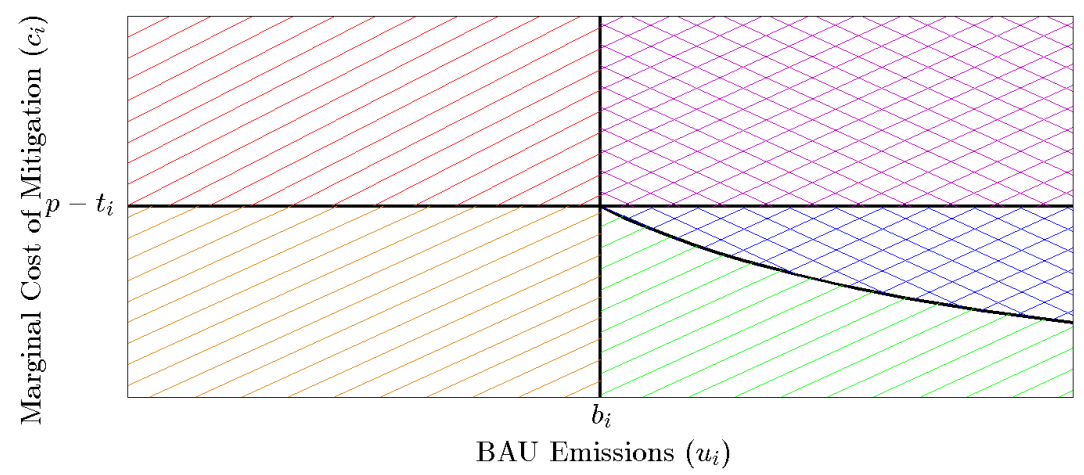

(b) Average Baseline

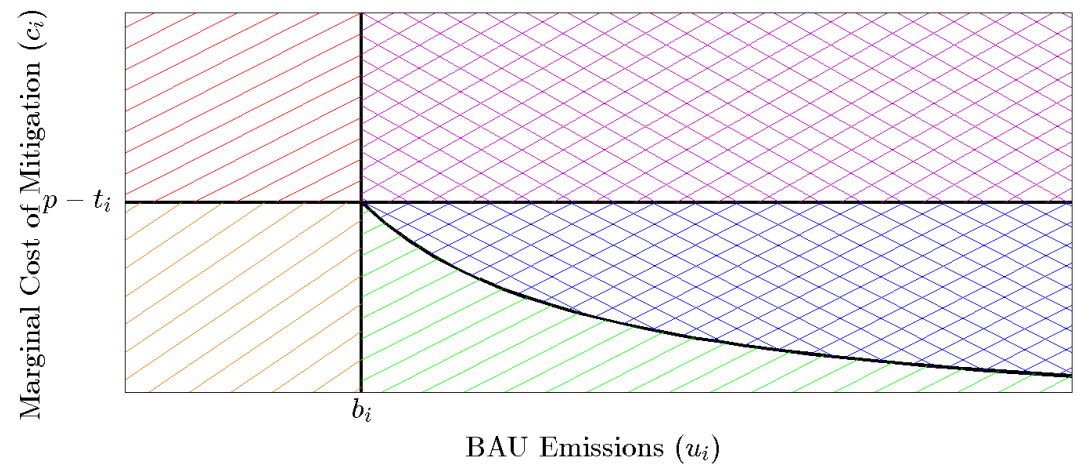

(c) Low (Conservative) Baseline

Supplementary Figure 2 $\mid$ The effect of baseline stringency on potential project decisions. The High Baseline case (a) is defined by a potential project being assigned a relatively generous baseline. This case represents a program establishing a lenient offsets protocol that does not account for BAU emissions uncertainty. The Low Baseline case (c) is defined by a potential project being assigned a relatively stringent baseline. This case represents a program establishing a conservative offsets protocol that accounts for BAU emissions uncertainty. 


\subsection{Net Carbon Price}

Supplementary Figure 3 displays versions of Figure 1 that illustrate the effect of different net carbon prices on project decisions. In Supplementary Figure 3, we vary the net carbon price faced by project $i, p-t_{i}$, from a low price in Panel (a) to high price in Panel (c). These panels emerge from either equilibrium market prices for offsets changing or from transaction costs to project $i$ changing. Moving from (a) to (c) represents an increase in the equilibrium offsets prices or a decrease in the transaction cost faced by project $i$, or a combination of the two. Several areas are influenced by the net carbon price. As the net carbon price increases, projects are more likely to be opted in and to mitigate emissions. This is represented by an expansion of the orange and green regions and a reduction of the red and purple regions. As a consequence, a given project is more likely to opt in and be under-credited. This effect illustrates one of our key results that as carbon prices increase (or as transaction costs fall), less stringent baselines are necessary to balance over-credited offsets with under-credited emissions reductions. 


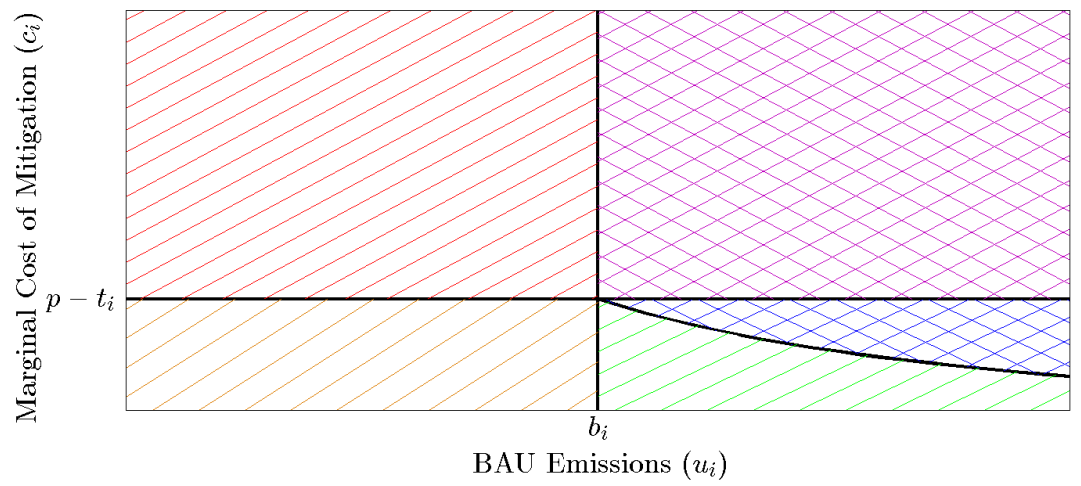

(a) Low Carbon Price and/or High Transaction Costs

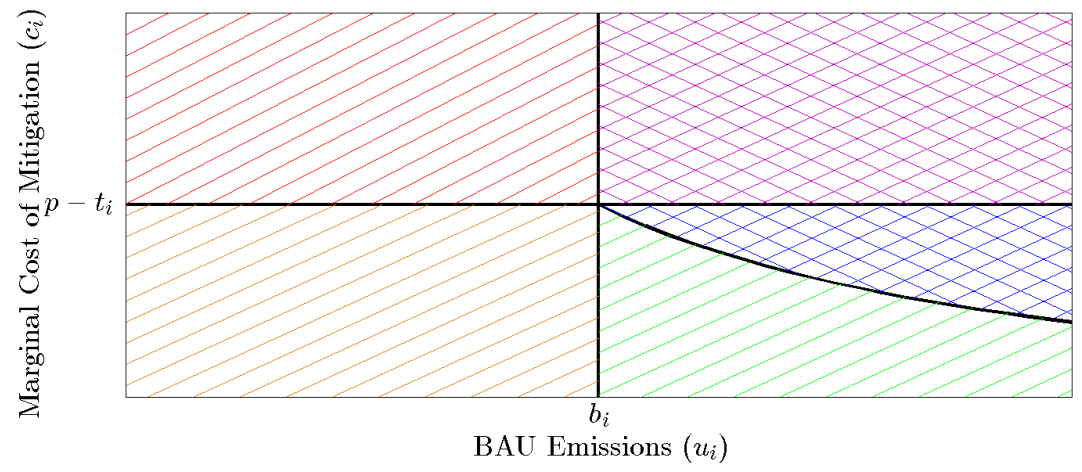

(b) Average Carbon Price and Average Transaction Costs

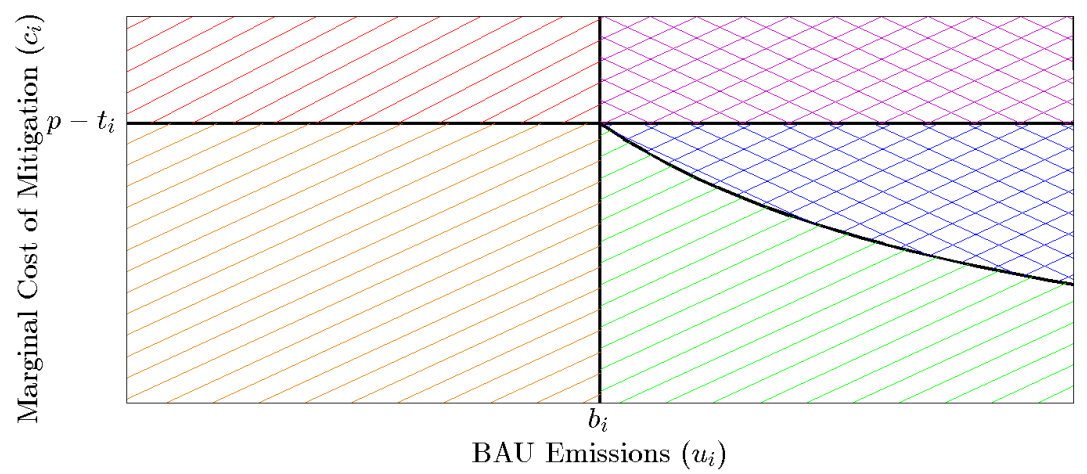

(c) High Carbon Price and/or Low Transaction Costs

Supplementary Figure 3 | The effect of equilibrium carbon prices and transaction costs on potential project decisions. The Low Carbon Price and/or High Transaction Costs case (a) is defined by a relatively low net carbon price $\left(p-t_{i}\right)$ faced by the project. This case represents predicted carbon prices for beginning years of a cap-and-trade program that has a declining emissions cap over time. The High Carbon Price and/or High Transaction Costs case (a) is defined by a relatively high net carbon price $\left(p-t_{i}\right)$ faced by the project. This case represents predicted carbon prices for later years of a cap-and-trade program that has a declining emissions cap over time. 


\section{Calibration}

The purpose of the numerical model is to yield generic insights that other researchers may apply to a range of climate mitigation programs. Even though our objective is to quantify general relationships, we choose a specific set of parameter values to calibrate the model and assign commonly used functional forms from the literature. Our central values represent emissions and mitigation costs of capped and uncapped sectors in the United States. The analytical equilibrium model is calibrated to observed emissions inventory data and Environmental Protection Agency (EPA) estimates of marginal mitigation costs and sequestration potential (EPA, 2009a,b, 2010).

\subsection{Capped Sector}

The capped sector marginal abatement cost function, $T A C^{\prime}(\cdot)$ is assumed to be increasing with a constant slope that matches processed simulation output of the EPA analysis of the U.S. Waxman-Markey bill (EPA, 2009a). We use processed simulation output from the Intertemporal General Equilibrium Model (IGEM) for the year 2016. We set the slope of the marginal abatement cost schedule equal to $2.83 \times 10^{-8} \$ /$ ton $^{2}$, so that

$$
T A C^{\prime}(q)=2.83 \times 10^{-8} q, \quad \text { (Supplementary Equation 21) }
$$

where $q$ denotes capped sector abatement in tons of $\mathrm{CO}_{2}$ equivalent. This implies that the demand for offsets is

$$
q^{*}(p)=\frac{p}{2.83 \times 10^{-8}}
$$

Integrating Supplementary Equation 21 and using the endpoint condition that $T A C(0)=0$ yields a capped sector total abatement cost $(T A C)$ schedule

$$
T A C(q)=1.415 \times 10^{-8} q^{2},
$$

(Supplementary Equation 23) 
where total costs are denoted in dollars. We assume that capped sector required abatement, denoted by $\bar{q}$, is equal to 500, 2,000 and 3,500 representing low, medium and high reduction targets that are specified in the Waxman-Markey bill (EPA, 2010). These are approximate reduction targets for years 2018, 2026 and 2034, respectively (EPA, 2010). To solve for total compliance costs when offsets are not allowed, we substitute the reduction target into Supplementary Equation 23.

\subsection{Uncapped Sector}

We set the number of potential projects equal to $n=1,000$. This value ensures that our fitted marginal cost of mitigation schedule closely approximates EPA marginal cost of mitigation data that we discuss below.

We calibrate uncapped sector parameters to data on potential carbon offsets sources in the United States. Sources include livestock management, crop management, afforestation, forest management and soil sequestration (EPA, 2009b). We calibrate the distribution of uncapped sector BAU emissions based on EPA projections of total annual net BAU emissions for the year 2020 (EPA, 2009b). These are defined as the sum of emissions and sequestration among offsets sources, which sum to $365 \mathrm{MMTCO}_{2}$ e per year of the program. Total Sequestration Potential is defined as the maximum quantity of sequestration that can occur among offsets sources. We obtain a value of $-1,027 \mathrm{MMTCO}_{2} \mathrm{e}$ by subtracting the EPA estimate of the supply of offsets at a carbon price of $\$ 211$ from Total Net BAU emissions (EPA, 2009b). This value represents an upper bound on the quantity of sequestration that can occur given marginal cost of mitigation estimates (EPA, 2009b).

We assume that the distributions for BAU emissions, sequestration potential and marginal costs of mitigation are uniform and independently distributed. Our results are not sensitive to correlation between BAU emissions and sequestration potential but are modestly sensitive to correlation between these variables and marginal costs of mitigation. We provide simulation results for cases when there is negative or positive correlation between these distributions (see Supplementary Tables 10, 11, 13, 14, 16, 17). After we draw BAU emissions and 
sequestration for each project, we assign marginal costs of mitigation to individual projects so that the resulting mitigation supply function approximates a polynomial fit of the points on the supply curves used in the EPA Waxman-Markey analysis for the year 2020 (EPA, 2009b). These points are plotted in Supplementary Figure 4 along with our resulting aggregate supply function.

We take the following steps to assign marginal costs of mitigation. First, we calculate a fifth-order polynomial that fits the points used in the EPA analysis (EPA, 2009b). These points appear in Supplementary Table 1 with the corresponding fitted polynomial.

\section{Supplementary Table 1 EPA Offsets Supply Estimates.}

\begin{tabular}{cc}
\hline \hline Offsets Price & Offsets Supply \\
\hline 0 & 0 \\
1 & 27.4 \\
5 & 156.4 \\
15 & 337.5 \\
30 & 560.9 \\
50 & 699.5 \\
\hline
\end{tabular}

Fitted polynomial

$$
\begin{aligned}
& \qquad F(p)=4.2 \times 10^{-2} p-2.2 \times 10^{-5} p^{2}+ \\
& \frac{1.5 \times 10^{-6} p^{3}-2.9 \times 10^{-9} p^{4}+2.0 \times 10^{-12} p^{5}}{\text { Offset prices are reported in dollars per ton of }} \\
& \mathrm{CO}_{2} \text { equivalent. Offsets supplies are reported } \\
& \text { as million metric tons of } \mathrm{CO}_{2} \text { equivalent. }
\end{aligned}
$$

Next we discretize the fitted polynomial into 101 offsets price-supply points starting at the offsets price of zero and ending at the offsets price of 100 . We then define an error function $\Delta(p)$ that measures the point-wise difference between the fitted polynomial and the 
supply of mitigation schedule from the 1,000 projects at equilibrium offsets price $p$ :

$$
\Delta(p)=F(p)-\sum_{i=1}^{n} f_{i}^{E}(p)
$$

Project marginal costs of mitigation are assigned through the solution to minimizing the sum of squared differences:

$$
\min _{c_{i}, i=1,2, \ldots, n}\left\{\sum_{P=0,1, \ldots, 100} \Delta(p)^{2}\right\}
$$

We use the simplex search method to solve Supplementary Equation 25 numerically. The solution to Supplementary Equation 25 yields an uncapped sector supply of mitigation function plotted in Supplementary Figure 4. As the figure shows, our algorithm generates a distribution of marginal costs of mitigation that yields an aggregate mitigation function that precisely fits the EPA data.

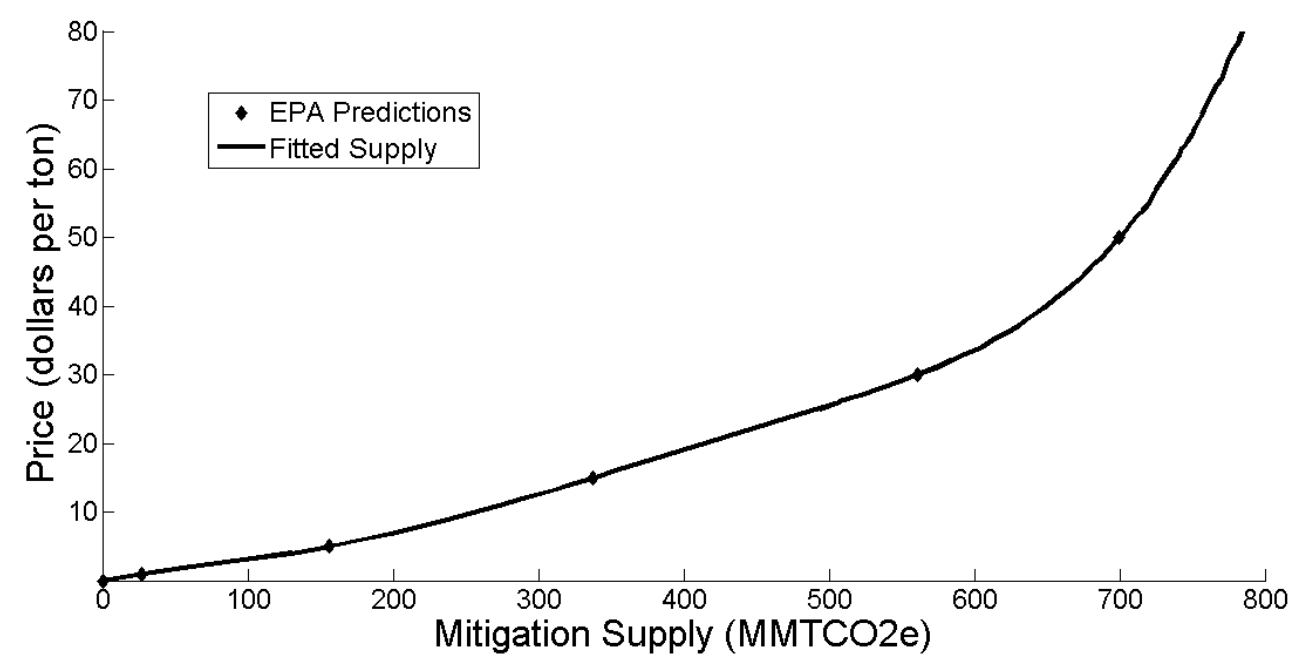

Supplementary Figure 4 | Calibrated uncapped sector supply of mitigation. The solid black line represents the marginal cost of mitigation curve $f_{i}^{E}(p)$. The diamonds represent EPA predictions for the supply of domestic offsets for the year 2020 (EPA, 2009b)

The standard deviation of the BAU emissions shocks is set to equal the expected value of BAU emissions. At this value the expected quantity of over-credited offsets equals 30 
percent of total offsets supply when baselines are set to equal predicted BAU emissions in an equilibrium with a carbon price equal to $\$ 25$ per ton of $\mathrm{CO}_{2} \mathrm{e}$. We base this calibration on evidence on a meta-study from the Clean Development Mechanism (CDM), the largest operating carbon offsets market (Schneider, 2007). We have three reasons for using estimates from the CDM. First, the CDM is the largest and most transparent carbon offsets program in existence. Second, many other offsets protocols are based on the CDM. Third, there does not exist a comprehensive analysis on the expected supply of over-credited offsets or the uncertainty in predicted BAU emissions for U.S. offsets providers. The study estimates that 20 percent offsets supply that is non-additional (Schneider, 2007). Our definition of over-credited offsets corresponds to how this study and others define non-additional offsets (Bushnell, 2012). We calibrate the model such that the benchmark supply of over-credited offsets is 30 percent to account for the fact that the composition of domestic projects in a U.S.-based program may be different than the composition of CDM projects (e.g., with the inclusion of U.S. forestry offsets). Moreover, we consider a wide range for the values of the standard deviation of the BAU emissions shocks in Figure 2 and a wider range in the sensitivity analysis to account for the uncertainty of this parameter. Larger standard deviations generally increase the share of over-credited offsets (see Supplementary Table 4).

We assign transaction costs to offsets projects in line with an analysis of Waxman-Markey by the Congressional Budget Office (Kile, 2009). The CBO adjusts the offsets supply schedule used by the EPA (Supplementary Figure 4) by adding a 5 dollar per ton of $\mathrm{CO}_{2}$ e transaction cost. This value lies within a range of transaction costs estimated in previous work.

Antinori and Sathaye (2007) compute transaction costs for 26 carbon offsets projects around the world. Their survey includes a variety of offsets project types, including forestry, energy efficiency, fuel switching, fuel capture and renewables. These projects operated between 1991 and 2005 and were verified and monitored through different offsets protocols, including the CDM, the Chicago Climate Exchange and Climate Trust. The authors find that transaction costs per ton of $\mathrm{CO}_{2} \mathrm{e}$ for the surveyed projects fall within the range of $\$ 0.03$ per ton of $\mathrm{CO}_{2} \mathrm{e}$ and $\$ 4.05$ per ton of $\mathrm{CO}_{2} \mathrm{e}$ with an average of $\$ 0.36$ per ton of $\mathrm{CO}_{2} \mathrm{e}$. 
Galik et al. (2012) estimate transaction costs for US-based forest carbon offsets projects. The authors used a detailed spreadsheet model that includes dis-aggregated forest types and 10 different regions. For all project types, transaction costs are estimated to be less than 25 percent of median implementation costs, which the authors define as the sum of production costs and transaction costs. In our model, if projects have transaction costs equal to 20 percent of implementation costs, the median transaction cost per ton of $\mathrm{CO}_{2} \mathrm{e}$ is 12.25 dollars, a value that is significantly higher than the range reported in Antinori and Sathaye.

We follow the CBO's approach by assigning a $\$ 5$ per ton of $\mathrm{CO}_{2}$ e transaction cost to all projects. Since there is substantial variability in this value, we perform sensitivity analysis across a wide range of values, including extreme cases of no transaction costs and $\$ 10$ per ton of $\mathrm{CO}_{2} \mathrm{e}$. We also consider cases in the sensitivity analysis where transaction costs equal a fixed percentage of a project's implementation costs and where implementation costs equal the sum of transaction costs and mitigation costs (see Supplementary Tables 25 and 26). These cases imply that total mitigation costs and transaction costs are positively correlated, a condition that is consistent with the estimation results reported in Galik et al.

Our model's calibrated parameter values appear in Supplementary Table 2. 


\section{Supplementary Table 2| Parameter Values.}

\begin{tabular}{|c|c|c|}
\hline Parameter description & Parameter & Value \\
\hline Lower Bound of BAU Emissions & $\underline{u}$ & 0 \\
\hline Upper Bound of BAU Emissions & $\bar{u}$ & 0.730 \\
\hline Lower Bound of Sequestration Potential & $\underline{s}$ & -2.054 \\
\hline Upper Bound of Sequestration Potential & $\bar{s}$ & 0 \\
\hline Standard Deviation of Emissions Shocks & $\sigma$ & 0.353 \\
\hline Transaction Costs & $t$ & $5 \$ /$ ton \\
\hline Slope of Capped Sector MAC & & $2.83 \times 10^{-8} \$ /$ ton $^{2}$ \\
\hline Capped Sector Reduction Targets & $q$ & $\{500 ; 2,000 ; 3,500\}$ \\
\hline
\end{tabular}




\section{Numerical Model Equilibrium and Output}

Given a sample of BAU emissions shocks, we solve for model outputs by computing a numerical equilibrium for the model. The numerical equilibrium is obtained by solving for an offsets price $p$ that satisfies Supplementary Equation 10, which we state again for ease of exposition:

$$
q^{*}(p)+f^{s}(p)=\bar{q} .
$$

In Supplementary Equation 26, $q^{*}(p)$ represents the capped sector quantity of abatement, $f^{s}(p)$ denotes the aggregate supply of offsets and $\bar{q}$ is the capped sector reduction target. Substituting the calibrated functions for equilibrium capped sector abatement, $q^{*}(p)$, and the supply of offsets, $f^{s}(p)$, yields

$$
\frac{p}{2.83 \times 10^{-8}}+\sum_{i=1}^{n} f_{i}^{s}\left(p, b_{i}, s_{i}, u_{i}, t_{i}\right)=\bar{q} .
$$

(Supplementary Equation 27)

We use a trust-region reflective search algorithm to solve for the equilibrium offsets price specified by Supplementary Equation 26. This algorithm searches for an offsets price that minimizes the distance between the sum of capped and uncapped sector abatement and the capped sector reduction target. The equilibrium offsets price is then fed into the equations for offsets supply, emissions and compliance costs. We repeat this process 2,000 times by drawing 2,000 different samples of BAU emissions shocks and solving for equilibrium prices and quantities. We average these results and report mean values for the relevant outputs. 


\section{Model Validation}

We validate the simulation model by comparing equilibrium prices and offsets quantities for different reduction targets to EPA analysis of the Waxman-Markey bill that uses the IGEM (EPA, 2010). IGEM is a deterministic, dynamic general equilibrium model that incorporates banking and borrowing behavior of regulated firms. The EPA assumes that all offsets supplied to the capped sector correspond to mitigation by the capped sector so that there are no over-credited offsets supplied and so that there is no quantity of under-credited emissions reductions. Our model, in contrast, includes supplies of over-credited offsets and has a positive quantity of under-credited emissions reductions. Furthermore, our model is static and does not include banking or borrowing. Nevertheless we demonstrate in this section that our model provides a good approximation to IGEM.

We simulate our model for six different reduction targets that correspond to reported abatement requirements in the bill between years 2015 and 2040. These targets range from $301 \mathrm{MMTCO}_{2} \mathrm{e}$ (year 2015) to 4,460 $\mathrm{MMTCO}_{2} \mathrm{e}$ (year 2040) and encompass our three reduction targets that we consider in the paper. The reduction targets requirements and simulation outputs appear in Supplementary Table 3. We report simulation outputs for the case where transaction costs equal zero to provide a more apples to apples comparison to the EPA simulation. 


\section{Supplementary Table 3 | Model validation.}

\begin{tabular}{|c|c|c|c|c|}
\hline \multirow{2}{*}{$\begin{array}{l}\text { Capped Sector } \\
\text { Reduction Target }\end{array}$} & \multicolumn{2}{|c|}{ Equilibrium Permit/Offsets Price } & \multicolumn{2}{|c|}{ Offsets supply } \\
\hline & IGEM & This Study & IGEM & This Study \\
\hline 301 (year 2015) & 21.10 & 2.83 & 312 & 54 \\
\hline 958 (year 2020) & 26.93 & 15.24 & 357 & 293 \\
\hline 1,828 (year 2025) & 34.36 & 32.95 & 423 & 508 \\
\hline 2,850 (year 2030) & 43.86 & 57.26 & 456 & 633 \\
\hline 3,663 (year 2035) & 55.98 & 79.56 & 534 & 685 \\
\hline 4,460 (year 2040) & 71.44 & 101.61 & 610 & 725 \\
\hline \multicolumn{5}{|c|}{$\begin{array}{l}\text { Results for the EPA IGEM model represent Scenario } 7 \text { - No international } \\
\text { offsets (EPA, 2010). The capped sector reduction target corresponds to the } \\
\text { required abatement for a given year that is based on business-as-usual emissions } \\
\text { projections. Equilibrium prices are reported in (year 2000) dollars. The capped } \\
\text { sector reduction target and the offsets supplies are reported in MMTCO } \mathrm{M}_{2} \text { e. The } \\
\text { offsets supplies provided do not include over-credited offsets. The simulation } \\
\text { results presented in this table do not include transaction costs to provide an } \\
\text { apples to apples comparison between our model and IGEM. }\end{array}$} \\
\hline
\end{tabular}

Equilibrium permit and offset prices are reported in (year 2000) dollars and the capped sector reduction target and the supply of offsets are reported in $\mathrm{MMTCO}_{2} \mathrm{e}$. For a proper comparison, we report offsets supply output that does not include over-credited offsets. Our model appears to fit the EPA IGEM simulations fairly well. A few differences between modeling assumptions are worth noting. First, the equilibrium prices are lower with a low reduction target and higher with a high reduction target in our model. For a reduction target of $958 \mathrm{MMTCO}_{2} \mathrm{e}$, our model predicts a permit price of $\$ 15.24$ while the IGEM model predicts a permit price of $\$ 26.93$. This occurs because our model does not incorporate the possibility for the capped sector to bank permits. The EPA predicts that the capped sector would significantly bank permits early to use later in the program. This mechanism has the effect of increasing the scarcity of permits in short-run compliance periods (which raises permit and offset prices) while lowering the scarcity of permits in long-run compliance 
periods (which lowers permit and offset prices). As a result, the EPA analysis has a flatter trajectory of permit prices. Furthermore, the EPA projects that capped firms would stop banking around 2025 and 2030, corresponding to the capped sector reduction targets that show good model fits between our model and the EPA model. In particular, with a capped sector reduction target of 1,828 , the equilibrium permit price predicted by the IGEM model is $\$ 34.36$ compared to $\$ 32.95$ in our model. Second, we incorporate a supply of over-credited offsets in our model while the EPA does not. For low capped sector reduction targets, the equilibrium price of offsets will be low, which means that a majority of the supply of offsets is over-credited. The large supply has the effect of depressing the equilibrium price of offsets. This effect is seen by recognizing that the supply of over-credited offsets is $145 \mathrm{MMCO}_{2} \mathrm{e}$, or about three times as large as the supply of exact offsets when the reduction target is 301 $\mathrm{MMTCO}_{2} \mathrm{e}$. 


\section{Sensitivity Analysis}

We perform sensitivity analysis by examining the key results over a wide range of parameter values. In particular, we vary the uncertainty in predicted BAU emissions, the marginal cost of mitigation from uncapped projects, the correlation between BAU emissions and sequestration potential, the correlation between BAU emissions and marginal costs of mitigation, the correlation between sequestration potential and marginal costs of mitigation, systemic bias in estimating predicted BAU emissions, transaction costs, and transaction costs as a proportion of total implementation costs. Supplementary Tables 4, 7, 10, 13, 16, 19, 22 and 25 report the ratio of under-credited emissions reductions to over-credited offsets for ranges of parameters. Supplementary Tables 5, 8, 11, 14, 17, 20, 23 and 26 report how offsets supply is sensitive to various parameters across the different baseline protocols. These tables report offsets supply relative to the benchmark model output. Supplementary Tables 6, $9,12,15,18,21,24$ and 27 report how cost savings of including offsets in cap-and-trade programs are sensitive to various parameters across the different baseline protocols. These tables report offsets supply relative to the benchmark model output. The following cases

include parameters that are varied around our benchmark assumptions where the capped sector reduction target equals 2,000 $\mathrm{MMTCO}_{2} \mathrm{e}$. 


\subsection{Standard Deviation of Emissions Shocks}

The ratio of under-credited emissions reductions to over-credited offsets appears to be sensitive to our assumption on the uncertainty in predicted BAU emissions (Supplementary Table 4). When the standard deviation of emissions shocks is high $(\sigma=2 E[u])$, the ratio falls below one when baselines are set to equal 60 percent of predicted BAU emissions. In contrast, when the standard deviation of emissions shocks is low $(\sigma=0.5 E[u])$, the ratio lies above one when baselines are set to 80 percent of predicted BAU emissions. Lower uncertainty requires less stringent baselines for all projects as fewer projects earn over-credited offsets.

Total offsets supply is not sacrificed when the standard deviation of emissions shocks is large (Supplementary Table 5). When the standard deviation is two times as large as it is in our benchmark model, total offsets supply declines by only about 10 percent under the maintain environmental integrity protocol, or about $55 \mathrm{MMTCO}_{2} \mathrm{e}$. This result suggests that even when there is significant uncertainty in BAU emissions, maintaining environmental integrity by adjusting baselines down can still be achieved without sacrificing a significant supply of offsets.

Supplementary Table 6 reports the sensitivity of cost savings from including offsets in capand-trade programs as we vary the standard deviation of emissions shocks. Increasing the standard deviation of emissions shocks increases the difference in cost savings between the protocols. Cost savings increase by about 18 percent under the predicted BAU emissions protocol while they fall by about 7 percent under the maintain environmental integrity protocol when the standard deviation is doubled to $\sigma=2 E[u]$. There are two effects that lead to the larger difference. First, a larger standard deviation increases the quantity of overcredited offsets, which has the effect of lowering compliance costs when offsets are allowed. This is seen by the increase in compliance costs savings for the predicted BAU emissions

protocol as $\sigma$ increases. Second, the quantity of under-credited emissions reductions falls as $\sigma$ increases, which requires setting more stringent baselines for all projects. More stringent baselines crowd out the supply of over-credited and exact offsets which, increases compliance costs. 
Supplementary Table $4 \mid$ Ratio of under-credited emissions reductions to over-credited offsets: Varying the standard deviation of emissions shocks.

\begin{tabular}{lcccccc}
\hline \hline & & \multicolumn{5}{c}{ Baseline Relative to Predicted BAU Emissions } \\
& $20 \%$ & $40 \%$ & $60 \%$ & $80 \%$ & $100 \%$ \\
\hline & $E[u]$ & 46.10 & 7.12 & 1.70 & 0.61 & 0.28 \\
\cline { 2 - 7 } & $0.5 E[u]$ & 201.95 & 29.19 & 6.06 & 1.31 & 0.35 \\
$\begin{array}{l}\text { Standard Deviation of } \\
\text { Emissions Shocks }(\sigma)\end{array}$ & $0.75 E[u]$ & 84.44 & 13.04 & 2.87 & 0.81 & 0.33 \\
& $1.5 E[u]$ & 19.17 & 2.90 & 0.89 & 0.39 & 0.22 \\
& $2 E[u]$ & 9.27 & 1.48 & 0.52 & 0.27 & 0.17 \\
\hline
\end{tabular}

A ratio above 1 implies that the quantity of under-credited emissions reductions exceeds the supply of over-credited offsets. We assume that the standard deviation of emissions shocks is equal to the expected value of BAU emissions $(\sigma=E[u])$ in the benchmark model. 
Supplementary Table $5 \mid$ The effect of alternative baseline protocols on offsets supply: Varying the standard deviation of emissions shocks.

(a) Exact Offsets Supply

\begin{tabular}{lcccc}
\hline \hline & & $\begin{array}{c}\text { Predicted } \\
\text { BAU Emissions }\end{array}$ & $\begin{array}{c}\text { Minimize Supply of } \\
\text { Over-Credited Offsets }\end{array}$ & $\begin{array}{c}\text { Maintain Environmental } \\
\text { Integrity }\end{array}$ \\
\hline & $E[u]$ & 505 & 338 & 497 \\
\cline { 2 - 5 } & $0.5 E[u]$ & $+9.11 \%$ & $+10.65 \%$ & $+9.66 \%$ \\
$\begin{array}{l}\text { Standard } \\
\text { Deviation of }\end{array}$ & & & $+5.03 \%$ & $+4.63 \%$ \\
$\begin{array}{l}\text { Emissions Shocks } \\
(\sigma)\end{array}$ & $0.75 E[u]$ & $+4.36 \%$ & $-8.28 \%$ & $-9.05 \%$ \\
& $1.5 E[u]$ & $-8.12 \%$ & $-13.31 \%$ & $-15.29 \%$ \\
\hline
\end{tabular}

(b) Total Offsets Supply

\begin{tabular}{lcccc}
\hline \hline & & $\begin{array}{c}\text { Predicted } \\
\text { BAU Emissions }\end{array}$ & $\begin{array}{c}\text { Minimize Supply of } \\
\text { Over-Credited Offsets }\end{array}$ & $\begin{array}{c}\text { Maintain Environmental } \\
\text { Integrity }\end{array}$ \\
\hline & $E[u]$ & 652 & 338 & 556 \\
\cline { 2 - 5 } & $0.5 E[u]$ & $-4.29 \%$ & $+10.69 \%$ & $+4.86 \%$ \\
$\begin{array}{l}\text { Standard } \\
\text { Deviation of }\end{array}$ & & & $+5.03 \%$ & $+2.70 \%$ \\
$(\sigma)$ & $0.75 E[u]$ & $-2.45 \%$ & $-8.28 \%$ & $-7.01 \%$ \\
& $1.5 E[u]$ & $+4.75 \%$ & $-13.61 \%$ & $-10.43 \%$ \\
\hline
\end{tabular}

Exact offsets supply represents the quantity of offsets that corresponds to mitigation from uncapped sector projects. Exact and total offsets supplies are reported in $\mathrm{MMTCO}_{2} \mathrm{e}$ for the benchmark model $(\sigma=E[u])$. The remaining cases are reported relative to the benchmark case. 
Supplementary Table 6 | The effect of alternative baseline protocols on cost savings from including offsets in cap-and-trade programs: Varying the standard deviation of emissions shocks.

\begin{tabular}{|c|c|c|c|c|}
\hline & & $\begin{array}{c}\text { Predicted } \\
\text { BAU Emissions }\end{array}$ & $\begin{array}{c}\text { Minimize Supply of } \\
\text { Over-Credited Offsets }\end{array}$ & $\begin{array}{l}\text { Maintain Environmental } \\
\text { Integrity }\end{array}$ \\
\hline \multirow{5}{*}{$\begin{array}{l}\text { Standard } \\
\text { Deviation of } \\
\text { Emissions } \\
\text { Shocks }(\sigma)\end{array}$} & $E[u]$ & 20,394 & 9,849 & 16,872 \\
\hline & $0.5 E[u]$ & $-7.97 \%$ & $+10.19 \%$ & $+2.15 \%$ \\
\hline & $0.75 E[u]$ & $-3.95 \%$ & $+4.77 \%$ & $+1.27 \%$ \\
\hline & $1.5 E[u]$ & $+8.73 \%$ & $-7.68 \%$ & $-4.98 \%$ \\
\hline & $2 E[u]$ & $+17.92 \%$ & $-12.20 \%$ & $-6.94 \%$ \\
\hline
\end{tabular}

Cost savings are relative to a program that does not allow offsets. These savings are reported in millions of dollars for the benchmark model $(\sigma=E[u])$. The remaining cases are reported relative to the benchmark case. 


\subsection{Supply of Mitigation}

We varied the supply of mitigation schedule between one quarter and four times the size of our benchmark model to see how the ratio of under-credited emissions reductions to over-credited offsets depends on the scale of mitigation opportunities in the uncapped sector. Each case represents a scenario where we scale the supply of mitigation schedule at every given offsets price by the percentage denoted in Supplementary Tables 7 and 8 . Supplementary Table 7 reports the ratio of under-credited emissions reductions to over-credited offsets. The case where we scale the supply of mitigation to 400 percent of the benchmark model represents a program that would incorporate a significantly larger supply of offsets, i.e., international offsets. A greater supply of offsets increases the ratio of under-credited emissions reductions to over-credited offsets. Increasing the supply of mitigation to 200 percent of the benchmark model increases the ratio from 0.61 to 0.96 when baselines are set to 80 percent of predicted BAU emissions. This effect can be explained by the fact that the average project has lower mitigation costs when the scope of offsets supply is broader, which encourages greater project participation to mitigate emissions. This has the effect of increasing the quantity of undercredited emissions reductions.

Across all protocols, increasing the supply of mitigation dramatically increases exact and total offsets supply (Supplementary Table 8). Doubling the supply of mitigation increases total offsets supply by between 44 percent and 76 percent. Moreover, increasing the supply of mitigation boosts offsets supply relatively more under the environmental integrity protocol than it does under then predicted BAU emissions protocol. Quadrupling the supply of mitigation doubles total offsets supply under the predicted BAU emissions protocol while it increases offsets supply by 125 percent under the maintain environmental integrity protocol. This is because increasing the supply of mitigation only increases the supply of exact offsets while not affecting the supply of over-credited offsets. As a result, as cheaper mitigation opportunities become available, the supplies under the different protocols become relatively closer.

Supplementary Table 9 reports the sensitivity of cost savings from including offsets in 
cap-and-trade programs as we vary the supply of mitigation. There are two insights from Supplementary Table 9. First, as the supply of mitigation increases, the cost savings from including offsets in cap-and-trade programs increase. Doubling the supply of offsets increases the cost savings by roughly 50 percent across the three protocols. This is because with a larger supply come cheaper reductions for a given offsets price. Second, as the supply of mitigation increases, the difference in cost savings between the protocols declines. This is because with a larger supply of offsets, there will be cheaper mitigation opportunities. As a result, the average project is more likely to be opted in even in the face of a baseline below its BAU, creating more under-credited emissions reductions. Hence, baselines can be made less stringent to maintain environmental integrity, leading to increased compliance cost savings. 
Supplementary Table 7 | Ratio of under-credited emissions reductions to over-credited offsets: Varying the supply of mitigation.

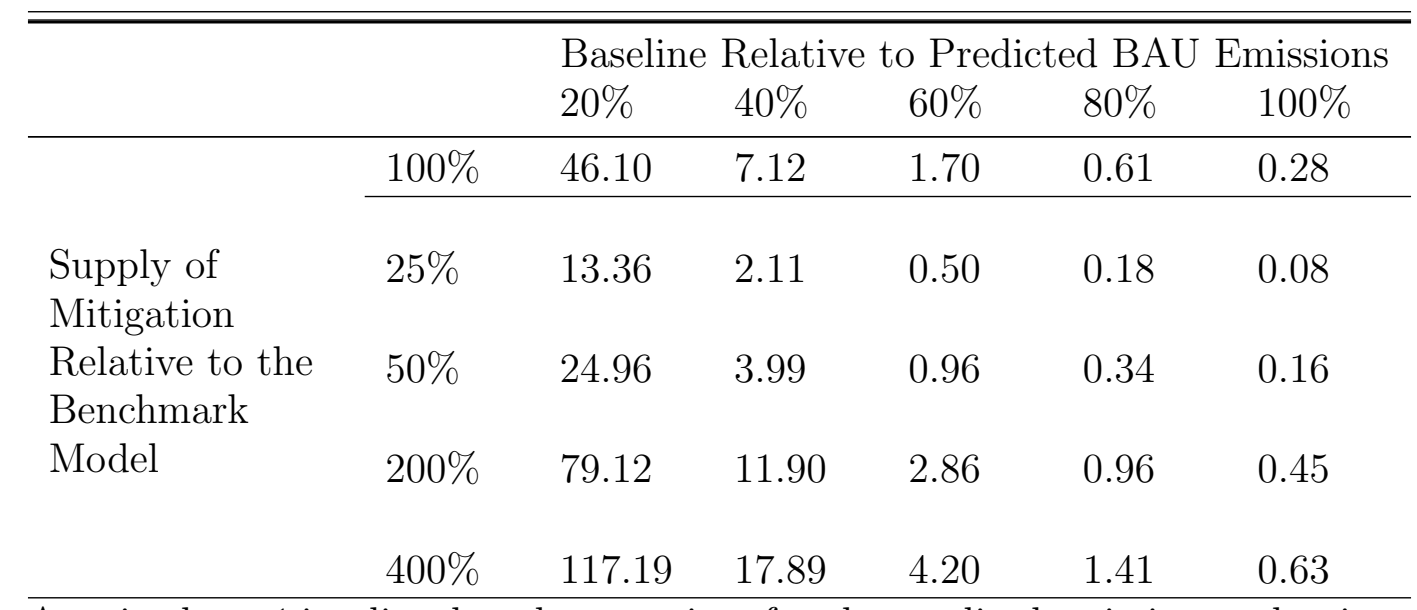

$\overline{\text { A ratio above } 1 \text { implies that the quantity of under-credited emissions reductions }}$ exceeds the supply of over-credited offsets. We vary the supply of mitigation schedule relative to the benchmark model by shifting the supply schedule. A proportion less than 100 percent implies a lower (steeper) supply of offsets schedule. Proportions above 100 percent represent cases where international offsets are allowed to be used by the capped sector. Our benchmark model is calibrated to fit EPA predictions of marginal costs of mitigation (see Supplementary Figure 4). 
Supplementary Table 8 | The effect of alternative baseline protocols on offsets supply: Varying the supply of mitigation.

(a) Exact Offsets Supply

\begin{tabular}{lcccc}
\hline \hline & & $\begin{array}{c}\text { Predicted } \\
\text { BAU Emissions }\end{array}$ & $\begin{array}{c}\text { Minimize Supply of } \\
\text { Over-Credited Offsets }\end{array}$ & $\begin{array}{c}\text { Maintain Environmental } \\
\text { Integrity }\end{array}$ \\
\hline & $100 \%$ & 505 & 338 & 497 \\
\cline { 2 - 5 } & $25 \%$ & $-71.29 \%$ & $-72.49 \%$ & $-73.24 \%$ \\
$\begin{array}{l}\text { Supply of } \\
\text { Mitigation }\end{array}$ & & & $-47.04 \%$ & $-47.08 \%$ \\
$\begin{array}{l}\text { Relative to } \\
\text { Benchmark }\end{array}$ & $50 \%$ & $-44.95 \%$ & $+76.33 \%$ & $+63.98 \%$ \\
Model & $200 \%$ & $+58.22 \%$ & +140.53 & $+131.79 \%$ \\
& $400 \%$ & $+125.94 \%$ & & \\
\hline
\end{tabular}

(b) Total Offsets Supply

\begin{tabular}{lcccc}
\hline \hline & & $\begin{array}{c}\text { Predicted } \\
\text { BAU Emissions }\end{array}$ & $\begin{array}{c}\text { Minimize Supply of } \\
\text { Over-Credited Offsets }\end{array}$ & $\begin{array}{c}\text { Maintain Environmental } \\
\text { Integrity }\end{array}$ \\
\hline & $100 \%$ & 652 & 338 & 556 \\
\cline { 2 - 5 } $\begin{array}{l}\text { Supply of } \\
\text { Mitigation }\end{array}$ & $25 \%$ & $-55.37 \%$ & $-72.49 \%$ & $-71.94 \%$ \\
$\begin{array}{l}\text { Relative to } \\
\text { Benchmark }\end{array}$ & $50 \%$ & $-34.97 \%$ & $-47.04 \%$ & $-46.22 \%$ \\
Model & $200 \%$ & $+44.94 \%$ & $+76.33 \%$ & $+61.51 \%$ \\
& $400 \%$ & $+97.39 \%$ & $+140.53 \%$ & $+125.00 \%$ \\
\hline
\end{tabular}

Exact offsets supply represents the quantity of offsets that correspond to mitigation from uncapped sector projects. Exact and total offsets supplies are reported in $\mathrm{MMTCO}_{2} \mathrm{e}$ for the benchmark model. The remaining cases are reported relative to the benchmark case. 
Supplementary Table 9 | The effect of alternative baseline protocols on cost savings from including carbon offsets in cap-and-trade programs: Varying the supply of mitigation.

\begin{tabular}{lcccc}
\hline \hline & $\begin{array}{c}\text { Predicted } \\
\text { BAU Emissions }\end{array}$ & $\begin{array}{c}\text { Minimize Supply of } \\
\text { Over-Credited Offsets }\end{array}$ & $\begin{array}{c}\text { Maintain Environmental } \\
\text { Integrity }\end{array}$ \\
\cline { 2 - 5 } & $100 \%$ & 20,394 & 9,849 & 16,872 \\
$\begin{array}{l}\text { Supply of } \\
\text { Mitigation }\end{array}$ & $25 \%$ & $-44.31 \%$ & $-71.53 \%$ & $-69.07 \%$ \\
$\begin{array}{l}\text { Relative to } \\
\text { Benchmark }\end{array}$ & $50 \%$ & $-27.29 \%$ & $-45.55 \%$ & $-43.02 \%$ \\
$\begin{array}{l}\text { Model } \\
\text { Sod }\end{array}$ & $200 \%$ & $+39.01 \%$ & $+70.23 \%$ & $+54.06 \%$ \\
& $400 \%$ & $+72.55 \%$ & $+154.38 \%$ & $+102.75 \%$ \\
\hline
\end{tabular}

Cost savings are relative to a program that does not allow offsets. These savings are reported in millions of dollars for the benchmark model. The remaining cases are reported relative to the benchmark case. 


\subsection{Correlation Between BAU Emissions and Sequestration Potential}

We vary the correlation between BAU emissions and sequestration potential from significantly negative correlation $\left(\rho_{u, s}=-0.8\right)$ to significantly positive correlation $\left(\rho_{u, s}=\right.$ 0.8). Supplementary Tables 10 and 11 report the ratio of under-credited emissions reductions to over-credited offsets and offsets supply for this range of correlation. The reported results suggest that the model output is insensitive to the correlation between these two random variables. With a correlation of $\rho=-0.8$ and baselines set to equal 80 percent of predicted BAU emissions, the ratio of under-credited emissions reductions to over-credited offsets is 0.57 . When the correlation is $\rho=0.8$, the ratio slightly rises to 0.63 (Supplementary Table 10).

Supplementary Table 12 reports the sensitivity of cost savings from including offsets in cap-and-trade programs as we vary the correlation between BAU emissions and sequestration

potential. Again we see that the correlation between these two variables has a negligible effect on the model outputs. Under the predicted BAU emissions protocol, compliance costs savings range from an increase of 0.55 percent $\left(\rho_{u, s}=-0.8\right)$ to a decrease of 0.37 percent $\left(\rho_{u, s}=0.8\right)$. 
Supplementary Table $10 \mid$ Ratio of under-credited emissions reductions to over-credited offsets: Varying the correlation between BAU emissions and sequestration potential.

\begin{tabular}{lcccccc}
\hline \hline & \multicolumn{5}{c}{ Baseline Relative to Predicted BAU Emissions } \\
& $20 \%$ & $40 \%$ & $60 \%$ & $80 \%$ & $100 \%$ \\
\hline & 0 & 46.10 & 7.12 & 1.70 & 0.61 & 0.28 \\
\cline { 2 - 7 } & -0.8 & 47.65 & 6.93 & 1.63 & 0.57 & 0.27 \\
$\begin{array}{l}\text { Correlation } \\
\text { Coefficient }\left(\rho_{u, s}\right)\end{array}$ & -0.4 & 46.94 & 7.12 & 1.71 & 0.58 & 0.27 \\
& 0.4 & 46.02 & 7.32 & 1.77 & 0.62 & 0.29 \\
& & & & & & \\
& 0.8 & 45.60 & 7.28 & 1.83 & 0.63 & 0.30 \\
\hline
\end{tabular}

A ratio above 1 implies that the quantity of under-credited emissions reductions exceeds the supply of over-credited offsets. We assume that there is no correlation between BAU emissions and sequestration potential $\left(\rho_{u, s}=0\right)$ in the benchmark model. 
Supplementary Table 11. The effect of alternative baseline protocols on offsets supply: Varying the correlation between BAU emissions and sequestration potential.

(a) Exact Offsets Supply

\begin{tabular}{lcccc}
\hline & & $\begin{array}{c}\text { Predicted } \\
\text { BAU Emissions }\end{array}$ & $\begin{array}{c}\text { Minimize Supply of } \\
\text { Over-Credited Offsets }\end{array}$ & $\begin{array}{c}\text { Maintain Environmental } \\
\text { Integrity }\end{array}$ \\
\hline & 0 & 505 & 338 & 497 \\
\cline { 2 - 5 } & -0.8 & $+0.59 \%$ & $+6.51 \%$ & $+0.60 \%$ \\
Correlation & & & $+2.96 \%$ & $+0.20 \%$ \\
& 0.4 & $-0.20 \%$ & $-2.96 \%$ & $-0.40 \%$ \\
& 0.8 & $-0.59 \%$ & $-6.21 \%$ & $-0.80 \%$ \\
\hline
\end{tabular}

(b) Total Offsets Supply

\begin{tabular}{lcccc}
\hline \hline & & $\begin{array}{c}\text { Predicted } \\
\text { BAU Emissions }\end{array}$ & $\begin{array}{c}\text { Minimize Supply of } \\
\text { Over-Credited Offsets }\end{array}$ & $\begin{array}{c}\text { Maintain Environmental } \\
\text { Integrity }\end{array}$ \\
\cline { 2 - 5 } & -0 & 652 & 338 & 556 \\
\hline Correlation & $+0.31 \%$ & $+6.51 \%$ & $-0.18 \%$ \\
Coefficient $\left(\rho_{u, s}\right)$ & -0.4 & $+0.15 \%$ & $+2.96 \%$ & $-0.18 \%$ \\
& 0.4 & $-0.31 \%$ & $-2.96 \%$ & $0.00 \%$ \\
& 0.8 & $-0.61 \%$ & $-6.21 \%$ & $0.00 \%$ \\
\hline
\end{tabular}

Exact offsets supply represents the quantity of offsets that correspond to mitigation from uncapped sector projects. Exact and total offsets supplies are reported in $\mathrm{MMTCO}_{2} \mathrm{e}$ for the benchmark model. The remaining cases are reported relative to the benchmark case. 
Supplementary Table 12 The effect of alternative baseline protocols on cost savings from including carbon offsets in cap-and-trade programs: Varying the correlation between BAU emissions and sequestration potential.

\begin{tabular}{lcccc}
\hline & & $\begin{array}{c}\text { Predicted } \\
\text { BAU Emissions }\end{array}$ & $\begin{array}{c}\text { Minimize Supply of } \\
\text { Over-Credited Offsets }\end{array}$ & $\begin{array}{c}\text { Maintain Environmental } \\
\text { Integrity }\end{array}$ \\
& -0.8 & $+0.55 \%$ & 9,849 & 16,872 \\
\cline { 2 - 5 } $\begin{array}{l}\text { Correlation } \\
\begin{array}{l}\text { Coefficient } \\
\left(\rho_{u, s}\right)\end{array}\end{array}$ & -0.4 & $+0.46 \%$ & $+5.19 \%$ & $-0.74 \%$ \\
& 0.4 & $-0.21 \%$ & $+2.52 \%$ & $-0.79 \%$ \\
& 0.8 & $-0.37 \%$ & $-2.68 \%$ & $+0.07 \%$ \\
& & & \\
& 0.5 & $-5.72 \%$ & $+0.46 \%$ \\
\hline
\end{tabular}

Cost savings are relative to a program that does not allow offsets. These savings are reported in millions of dollars for the benchmark model. The remaining cases are reported relative to the benchmark case. 


\subsection{Correlation Between BAU Emissions and Marginal Costs of Mitigation}

We vary the correlation between BAU emissions and marginal costs of mitigation from highly negative correlation $\left(\rho_{u, c}=-0.8\right)$ to highly positive correlation $\left(\rho_{u, c}=0.8\right)$. Supplementary Tables 13 and 14 report the ratio of under-credited emissions reductions to over-credited offsets and offsets supply for this range of correlation.

With baselines set equal to predicted BAU emissions, a greater correlation between BAU emissions and marginal costs of mitigation increases the relative quantity of under-credited emissions reductions. Increasing the correlation from $\rho_{u, c}=0$ to $\rho_{u, c}=0.8$ increases the ratio of under-credited emissions reductions to over-credited offsets from 0.28 to 0.32 . A positive correlation between these two variables implies that projects with high marginal mitigation costs have high BAU emissions. Projects with high mitigation costs require a large mitigation potential to opt in if their baseline is below their BAU emissions. These projects, however, will have high mitigation potential because of the imposed correlation. As a result, more projects with baselines below their BAU emissions opt in, leading to a higher quantity of under-credited emissions reductions.

This effect, however, is dominated by a second effect when baselines are set below predicted BAU emissions. Projects with high mitigation costs do not opt in when baselines are set below predicted BAU emissions because the revenue incentive is no longer great enough. At the same time, projects with low marginal costs that have small BAU emissions (due to the imposed correlation) are likely to contribute fewer under-credited emissions reductions. This is because the difference between the baselines and the BAU emissions of these projects is likely to be small, since baselines are positively related to BAU emissions. In other words, a project with low BAU emissions has a lower potential for contributing under-credited emissions reductions. Since projects with low marginal costs are those that opt in, projects that mitigate emissions will provide less under-credited emissions reductions in this case.

Supplementary Table 15 reports the sensitivity of cost savings from including offsets in cap-and-trade programs as we vary the correlation between BAU emissions and marginal 
costs of mitigation. As the correlation between BAU emissions and marginal costs of mitigation increases, cost savings from allowing offsets fall across all three protocols. Under the maintain environmental integrity protocol, increasing the correlation from $\rho_{u, c}=0$ to $\rho_{u, c}=0.8$ reduces cost savings by about 27 percent. This is because projects with high mitigation costs are more likely to opt in while those with low mitigation costs are less likely to opt in when the correlation between BAU emissions and marginal costs of mitigation is positive. Increasing a project's BAU emissions raises its potential revenue from opting in with all other characteristics held constant. Assuming a positive correlation between BAU emissions and marginal costs of mitigation essentially sets BAU emissions low for projects with low marginal costs and sets BAU emissions high for projects with high marginal costs. Relative to our benchmark model where these variables are independent, assigning a positive correlation reduces the profit incentive for low marginal cost of mitigation projects while raising the profit incentive for high marginal cost of mitigation projects. As a result, we see that a positive correlation increases total mitigation costs of projects that opt in, thereby reducing cost savings from including offsets in the cap-and-trade program. 
Supplementary Table $13 \mid$ Ratio of under-credited emissions reductions to over-credited offsets: Varying the correlation between BAU emissions and marginal costs of mitigation.

\begin{tabular}{lcccccc}
\hline \hline & \multicolumn{6}{c}{ Baseline Relative to Predicted BAU Emissions } \\
& $20 \%$ & $40 \%$ & $60 \%$ & $80 \%$ & $100 \%$ \\
\hline & 0 & 46.10 & 7.12 & 1.70 & 0.61 & 0.28 \\
\cline { 2 - 7 } & -0.8 & 1273.79 & 44.75 & 3.86 & 0.70 & 0.24 \\
$\begin{array}{l}\text { Correlation } \\
\text { Coefficient }\left(\rho_{u, c}\right)\end{array}$ & -0.4 & 185.18 & 18.34 & 2.66 & 0.65 & 0.25 \\
& 0.4 & 10.75 & 2.94 & 1.03 & 0.59 & 0.28 \\
& 0.8 & 2.89 & 1.43 & 0.78 & 0.51 & 0.32 \\
\hline
\end{tabular}

$\overline{\text { A ratio above } 1 \text { implies that the quantity of under-credited emissions reductions }}$ exceeds the supply of over-credited offsets. We assume that there is no correlation between BAU emissions and sequestration potential $\left(\rho_{u, c}=0\right)$ in the benchmark model. 
Supplementary Table 14. The effect of alternative baseline protocols on offsets supply: Varying the correlation between BAU emissions and marginal costs of mitigation.

(a) Exact Offsets Supply

\begin{tabular}{lcccc}
\hline & & $\begin{array}{c}\text { Predicted } \\
\text { BAU Emissions }\end{array}$ & $\begin{array}{c}\text { Minimize Supply of } \\
\text { Over-Credited Offsets }\end{array}$ & $\begin{array}{c}\text { Maintain Environmental } \\
\text { Integrity }\end{array}$ \\
\hline & 0 & 505 & 338 & 497 \\
\cline { 2 - 5 } & -0.8 & $-11.49 \%$ & $-12.72 \%$ & $-10.66 \%$ \\
Correlation & & $-8.91 \%$ & $-3.55 \%$ & $-5.03 \%$ \\
& 0.4 & $+2.57 \%$ & $+0.89 \%$ & $+1.41 \%$ \\
& 0.8 & $+3.02 \%$ & $+1.98 \%$ & $+2.62 \%$ \\
\hline
\end{tabular}

(b) Total Offsets Supply

\begin{tabular}{lcccc}
\hline \hline & & $\begin{array}{c}\text { Predicted } \\
\text { BAU Emissions }\end{array}$ & $\begin{array}{c}\text { Minimize Supply of } \\
\text { Over-Credited Offsets }\end{array}$ & $\begin{array}{c}\text { Maintain Environmental } \\
\text { Integrity }\end{array}$ \\
\hline & 0 & 652 & 338 & 556 \\
\hline & -0.8 & $-7.52 \%$ & $-12.72 \%$ & $-14.93 \%$ \\
Correlation & & $-6.29 \%$ & $-3.55 \%$ & $-7.73 \%$ \\
Coefficient $\left(\rho_{u, c}\right)$ & -0.4 & & $+0.89 \%$ & $+4.50 \%$ \\
& 0.4 & $+0.46 \%$ & $+1.98 \%$ & $+10.61 \%$ \\
\hline
\end{tabular}

Exact offsets supply represents the quantity of offsets that correspond to mitigation from uncapped sector projects. Exact and total offsets supplies are reported in $\mathrm{MMTCO}_{2} \mathrm{e}$ for the benchmark model. The remaining cases are reported relative to the benchmark case. 
Supplementary Table 15 The effect of alternative baseline protocols on cost savings from including carbon offsets in cap-and-trade programs: Varying the correlation between BAU emissions and marginal costs of mitigation.

\begin{tabular}{lcccc}
\hline & & $\begin{array}{c}\text { Predicted } \\
\text { BAU Emissions }\end{array}$ & $\begin{array}{c}\text { Minimize Supply of } \\
\text { Over-Credited Offsets }\end{array}$ & $\begin{array}{c}\text { Maintain Environmental } \\
\text { Integrity }\end{array}$ \\
& -0.8 & $+48.57 \%$ & 9,849 & 16,872 \\
\cline { 2 - 5 } & & 20,394 & $+42.64 \%$ & $+39.75 \%$ \\
$\begin{array}{l}\text { Correlation } \\
\left(\rho_{u, c}\right)\end{array}$ & -0.4 & $+33.21 \%$ & $+30.27 \%$ & $+27.91 \%$ \\
& 0.4 & $-9.97 \%$ & $-21.19 \%$ & $-5.70 \%$ \\
& 0.8 & $-30.32 \%$ & $-47.77 \%$ & $-26.76 \%$ \\
\hline
\end{tabular}

Cost savings are relative to a program that does not allow offsets. These savings are reported in millions of dollars for the benchmark model. The remaining cases are reported relative to the benchmark case. 


\subsection{Correlation Between Sequestration Potential and Marginal Costs of Mitigation}

We vary the correlation between sequestration potential and marginal costs of mitigation from highly negative correlation $\left(\rho_{s, c}=-0.8\right)$ to highly positive correlation $\left(\rho_{s, c}=0.8\right)$. Supplementary Tables 16 and 17 report the ratio of under-credited emissions reductions to over-credited offsets and offsets supply for this range of correlation. The reported results suggest that the model is modestly sensitive to the correlation between these two random variables. With a correlation of $\rho_{s, c}=-0.8$ and baselines set to equal 80 percent of predicted BAU emissions, the ratio of under-credited emissions reductions to over-credited offsets is 0.92. When the correlation is $\rho_{s, c}=0.8$, the ratio falls to 0.50 (Supplementary Table 16). These results suggest that the greater the correlation between sequestration potential and mitigation costs, the lower the quantity of under-credited emissions reductions. A positive correlation between these two variables implies that projects with low marginal mitigation costs have a high sequestration potential. Projects with low mitigation costs are likely to opt in regardless of their sequestration potential. On the other hand, projects with high mitigation costs require a large mitigation potential to opt in if their baseline is below their BAU emissions. These projects, however, will have low mitigation potential because of the imposed correlation. As a result, fewer projects with baselines below their BAU emissions opt in, leading to a lower quantity of under-credited emissions reductions.

The reason that a negative correlation causes the proportion of under-credited emissions reductions to increase is opposite to the case of positive correlation. Projects with high marginal costs of mitigation are more likely to opt in because they are likely to have a large sequestration potential. As a result, those projects that are assigned a baseline below BAU emissions have a greater incentive to opt in and contribute under-credited emissions reductions. This can be seen in Supplementary Table 16. Increasing the correlation from $\rho_{s, c}=0$ to $\rho_{s, c}=0.8$ increases the ratio of under-credited emissions reductions to overcredited offsets from 1.70 to 2.78 when baselines are set to 60 percent of predicted BAU emissions. This relationship is further established in Table 17. For all three protocols, 
increasing the correlation between sequestration potential and marginal mitigation costs increases the quantity of offsets from projects mitigating emissions (Panel (a)) and total offsets supply (Panel (b)).

Supplementary Table 18 reports the sensitivity of cost savings from including offsets in cap-and-trade programs as we vary the correlation between sequestration potential and marginal costs of mitigation. As the correlation between sequestration potential and marginal costs of mitigation increases, cost savings from allowing offsets dramatically increase across all three protocols. Under the predicted BAU emissions protocol, increasing the correlation from $\rho_{s, c}=0$ to $\rho_{s, c}=0.8$ increases cost savings by about 50 percent. A positive correlation between sequestration potential and marginal costs of mitigation implies that projects with low marginal costs are likely to have very large, negative sequestration potential. The more negative the sequestration potential, the larger the offsets supply potential. As a consequence, assuming a positive correlation implies that very large projects will provide cheap mitigation opportunities while smaller projects are more expensive. Since the projects with low marginal costs of mitigation will opt in, a greater quantity of cheap offsets will be supplied. As a result, the cost savings from including offsets in cap-in-trade programs will be greater. 
Supplementary Table $16 \mid$ Ratio of under-credited emissions reductions to over-credited offsets: Varying the correlation between sequestration potential and marginal costs of mitigation.

\begin{tabular}{lcccccc}
\hline \hline & \multicolumn{5}{c}{ Baseline Relative to Predicted BAU Emissions } \\
& $20 \%$ & $40 \%$ & $60 \%$ & $80 \%$ & $100 \%$ \\
\hline & 0 & 46.10 & 7.12 & 1.70 & 0.61 & 0.28 \\
\cline { 2 - 6 } & -0.8 & 87.69 & 12.69 & 2.78 & 0.92 & 0.41 \\
$\begin{array}{l}\text { Correlation } \\
\text { Coefficient }\left(\rho_{s, c}\right)\end{array}$ & -0.4 & 71.71 & 10.44 & 2.49 & 0.84 & 0.38 \\
& 0.4 & 42.55 & 6.74 & 1.60 & 0.56 & 0.26 \\
& & & & & & \\
& 0.8 & 39.92 & 6.07 & 1.48 & 0.50 & 0.23 \\
\hline
\end{tabular}

A ratio above 1 implies that the quantity of under-credited emissions reductions exceeds the supply of over-credited offsets. We assume that there is no correlation between sequestration potential and marginal costs of mitigation $\left(\rho_{s, c}=0\right)$ in the benchmark model. 
Supplementary Table 17 | The effect of alternative baseline protocols on offsets supply: Varying the correlation between sequestration potential and marginal costs of mitigation.

(a) Exact Offsets Supply

\begin{tabular}{lcccc}
\hline & & $\begin{array}{c}\text { Predicted } \\
\text { BAU Emissions }\end{array}$ & $\begin{array}{c}\text { Minimize Supply of } \\
\text { Over-Credited Offsets }\end{array}$ & $\begin{array}{c}\text { Maintain Environmental } \\
\text { Integrity }\end{array}$ \\
\hline & 0 & 505 & 338 & 497 \\
\cline { 2 - 5 } & -0.8 & $-4.95 \%$ & $-45.27 \%$ & $-7.85 \%$ \\
Correlation & & $-1.98 \%$ & $-30.47 \%$ & $-3.62 \%$ \\
& 0.4 & $+11.68 \%$ & $+20.41 \%$ & $+11.67 \%$ \\
& 0.8 & $+12.87 \%$ & $+27.81 \%$ & $+13.68 \%$ \\
\hline
\end{tabular}

(b) Total Offsets Supply

\begin{tabular}{lcccc}
\hline \hline & & $\begin{array}{c}\text { Predicted } \\
\text { BAU Emissions }\end{array}$ & $\begin{array}{c}\text { Minimize Supply of } \\
\text { Over-Credited Offsets }\end{array}$ & $\begin{array}{c}\text { Maintain Environmental } \\
\text { Integrity }\end{array}$ \\
\hline & 0 & 652 & 338 & 556 \\
\hline & -0.8 & $-4.45 \%$ & $-45.27 \%$ & $-3.78 \%$ \\
Correlation & & $-1.69 \%$ & $-30.47 \%$ & $-1.98 \%$ \\
Coefficient $\left(\rho_{s, c}\right)$ & -0.4 & $+8.90 \%$ & $+20.41 \%$ & $+9.35 \%$ \\
& 0.4 & & & $+9.71 \%$ \\
\hline
\end{tabular}

Exact offsets supply represents the quantity of offsets that correspond to mitigation from uncapped sector projects. Exact and total offsets supplies are reported in $\mathrm{MMTCO}_{2} \mathrm{e}$ for the benchmark model. The remaining cases are reported relative to the benchmark case. 
Supplementary Table 18 The effect of alternative baseline protocols on cost savings from including offsets in cap-and-trade programs: Varying the correlation between sequestration potential and marginal costs of mitigation.

\begin{tabular}{lcccc}
\hline & & $\begin{array}{c}\text { Predicted } \\
\text { BAU Emissions }\end{array}$ & $\begin{array}{c}\text { Minimize Supply of } \\
\text { Over-Credited Offsets }\end{array}$ & $\begin{array}{c}\text { Maintain Environmental } \\
\text { Integrity }\end{array}$ \\
& -0.8 & $-57.57 \%$ & 9,849 & 16,872 \\
\cline { 2 - 5 } $\begin{array}{l}\text { Correlation } \\
\begin{array}{l}\text { Coefficient } \\
\left(\rho_{s, c}\right)\end{array}\end{array}$ & -0.4 & $-46.07 \%$ & $-66.43 \%$ & $-45.06 \%$ \\
& 0.4 & $+29.90 \%$ & $-64.00 \%$ & $-39.82 \%$ \\
& & & $+58.21 \%$ & $+28.45 \%$ \\
& 0.8 & $+50.11 \%$ & $+72.75 \%$ & $+58.70 \%$ \\
\hline
\end{tabular}

Cost savings are relative to a program that does not allow offsets. These savings are reported in millions of dollars in the benchmark model. The remaining cases are reported relative to the benchmark case. 


\subsection{Systematic Bias in Predicted BAU Emissions}

The benchmark model does not incorporate systematic bias, which is defined as the tendency to consistently over- or underestimate a true value. In this section we investigate the role that systematic bias in estimating predicted BAU emissions may play in the relative magnitude of under-credited emissions reductions. We model systematic bias by augmenting the baseline Supplementary Equation 5 with a bias parameter $\beta$ :

$$
b_{i}=\alpha \beta \tilde{u}_{i}
$$

(Supplementary Equation 28)

When there is no bias in estimating predicted BAU emissions, $\beta=1$ and we are back to our benchmark model. When predicted BAU emissions are consistently overestimated, $\beta>1$. When predicted BAU emissions are consistently underestimated, $\beta<1$. In the tables below we report the bias relative to the no bias case. For example, if $\beta=1.2$, we represent this bias as +20 percent.

We find that systematic bias has a significant impact on relative quantity of under-credited emissions reductions. When there is negative bias in predicting BAU emissions, the ratio of under-credited emissions reductions to over-credited offsets increases. The ratio is about twice as large when the bias is -20 percent compared to the benchmark setting of no bias (Supplementary Table 19). When there is positive bias, the ratio of under-credited emissions reductions to over-credited offsets decreases. The ratio is about half as large when the bias is +20 percent compared to the benchmark setting of no bias (Supplementary Table 19).

Incorporating bias into the analysis shifts the reference point of the baseline. When the bias is positive, the established baseline as a fraction of predicted BAU emissions is not as stringent as in the settings when no bias is present. This effect is quantified in Table 20. When baselines are set equal to predicted BAU emissions, total offsets supply dramatically

increases as the bias increases. This increase in offsets supply comes from a significant expansion of over-credited offsets. In fact, the supply of exact offsets contracts as the bias increases (Panel (a)). 
Supplementary Table 21 reports the sensitivity of cost savings from including offsets in cap-and-trade programs as we vary the systematic bias in estimating predicted BAU emissions. Cost savings from including offsets in cap-and-trade programs only appears sensitive to systematic bias in the predicted BAU emissions protocol. Increasing the bias to 20 percent increases cost savings by about 9 percent. This is because a positive bias creates a larger supply of over-credited offsets when baselines do not adjust. When baselines are adjusted in the minimize supply of over-credited offsets and maintain environmental integrity protocols, cost savings do not dramatically change with different versions of bias. This is because the baseline can be adjusted to account for any bias in estimating predicted BAU emissions. Any positive bias can be accounted for by lowering the baseline. Likewise, any negative bias can be accounted for by increasing the baseline. 
Supplementary Table 19 | Ratio of under-credited emissions reductions to over-credited offsets: Varying systematic bias in predicted BAU emissions.

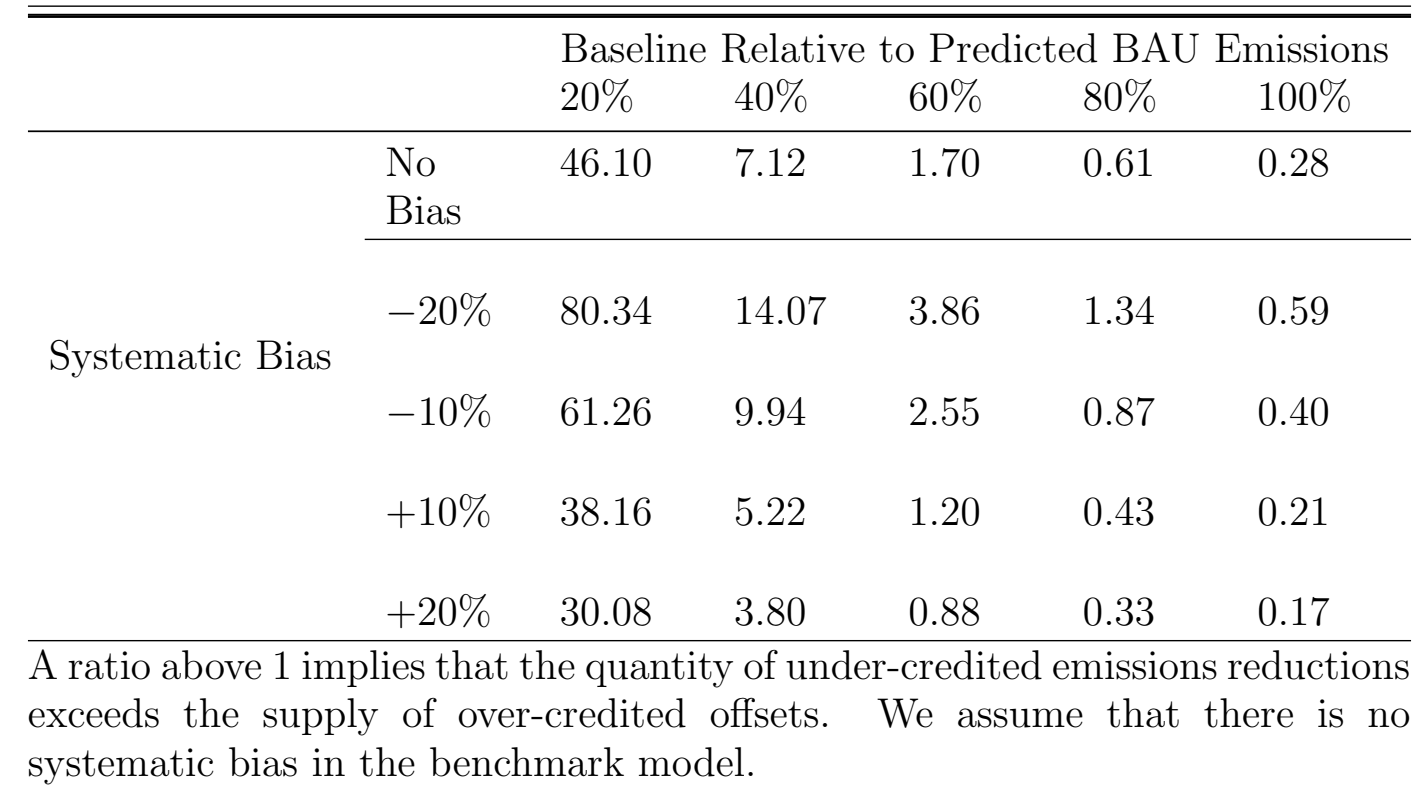


Supplementary Table 20 | The effect of alternative baseline protocols on offsets supply: Varying systematic bias in predicted BAU emissions.

(a) Exact Offsets Supply

\begin{tabular}{ccccc}
\hline \hline & $\begin{array}{c}\text { Predicted } \\
\text { BAU Emissions }\end{array}$ & $\begin{array}{c}\text { Minimize Supply of } \\
\text { Over-Credited Offsets }\end{array}$ & $\begin{array}{c}\text { Maintain Environmental } \\
\text { Integrity }\end{array}$ \\
\hline & No Bias & 505 & 338 & 497 \\
\cline { 2 - 5 } Systematic Bias & $-20 \%$ & $+0.79 \%$ & $0.00 \%$ & $+1.21 \%$ \\
& $-10 \%$ & $+0.40 \%$ & $0.00 \%$ & $+0.60 \%$ \\
& $+10 \%$ & $-0.99 \%$ & $0.00 \%$ & $-1.81 \%$ \\
& & & $0.00 \%$ & $-2.41 \%$ \\
\hline
\end{tabular}

(b) Total Offsets Supply

\begin{tabular}{ccccc}
\hline \hline & & $\begin{array}{c}\text { Predicted } \\
\text { BAU Emissions }\end{array}$ & $\begin{array}{c}\text { Minimize Supply of } \\
\text { Over-Credited Offsets }\end{array}$ & $\begin{array}{c}\text { Maintain Environmental } \\
\text { Integrity }\end{array}$ \\
\hline & No Bias & 652 & 338 & 556 \\
\cline { 2 - 5 } & $-20 \%$ & $-9.05 \%$ & $0.00 \%$ & $+0.72 \%$ \\
Systematic Bias & $-10 \%$ & $-4.75 \%$ & $0.00 \%$ & $0.00 \%$ \\
& $+10 \%$ & $+3.99 \%$ & $0.00 \%$ & $-3.60 \%$ \\
& $+20 \%$ & $+8.90 \%$ & $0.00 \%$ & $-1.62 \%$ \\
\hline
\end{tabular}

Exact offsets supply represents the quantity of offsets that correspond to mitigation from uncapped sector projects. Exact and total offsets supplies are reported in $\mathrm{MMTCO}_{2} \mathrm{e}$ for the benchmark model. The remaining cases are reported relative to the benchmark case. 
Supplementary Table 21 | The effect of alternative baseline protocols on cost savings from including offsets in cap-and-trade programs: Varying the systematic bias in estimating predicted BAU emissions.

\begin{tabular}{|c|c|c|c|c|}
\hline & & $\begin{array}{c}\text { Predicted } \\
\text { BAU Emissions }\end{array}$ & $\begin{array}{c}\text { Minimize Supply of } \\
\text { Over-Credited Offsets }\end{array}$ & $\begin{array}{c}\text { Maintain Environmental } \\
\text { Integrity }\end{array}$ \\
\hline \multirow{5}{*}{$\begin{array}{l}\text { Systematic } \\
\text { Bias }\end{array}$} & No Bias & 20,394 & 9,849 & 16,872 \\
\hline & $-20 \%$ & $-11.33 \%$ & $0.00 \%$ & $+0.14 \%$ \\
\hline & $-10 \%$ & $-5.49 \%$ & $0.00 \%$ & $0.00 \%$ \\
\hline & $+10 \%$ & $+5.74 \%$ & $0.00 \%$ & $-5.23 \%$ \\
\hline & $+20 \%$ & $+11.32 \%$ & $0.00 \%$ & $-1.86 \%$ \\
\hline
\end{tabular}

Cost savings are relative to a program that does not allow offsets. These savings are reported in millions of dollars for the benchmark model. The remaining cases are reported relative to the benchmark case. 


\subsection{Transaction Costs}

We vary the assumption of assigning a $\$ 5$ per ton of $\mathrm{CO}_{2} \mathrm{e}$ transaction cost to each project by considering a wide range of alternative values. All project types, including those that are over-credited and those that are under-credited, are less likely to be opted in when transaction costs are higher since the marginal revenue from supplying an offset is less. We find that higher transaction costs have a mild effect on the ratio of under-credited emissions reductions to over-credited offsets (Supplementary Table 22). Increasing the transaction cost per ton of offsets from $\$ 5$ per ton to $\$ 10$ per ton reduces the ratio from 0.28 to 0.24 when baselines equal predicted BAU emissions.

Larger transaction costs have the effect of reducing the supply of offsets and lowering the efficiency of incorporating offsets in cap-and-trade programs. We find that projects facing transaction costs are less likely to be opted in to the program. More specifically, we find that projects that supply over-credited offsets and those that generate under-credited emissions reductions are both less likely to participate. The sacrifice in total offsets supply by maintaining environmental integrity does not change much when transaction costs are incorporated (Supplementary Table 23).

Supplementary Table 24 reports the sensitivity of cost savings from including offsets in cap-and-trade programs as we vary the per ton of $\mathrm{CO}_{2}$ transaction cost. Increasing transaction costs lowers cost savings from including offsets in cap-and-trade programs. The effect, however, is mild. Doubling the per unit transaction cost from 5 dollars per ton to $\$ 10$ per ton reduces cost savings by about $\$ 3$ billion for each protocol, which is approximately a 15 percent reduction. The reason that cost savings are not more sensitive to higher transaction costs is primarily due to the equilibrium offsets price. In these sensitivity runs we assume a medium benchmark capped sector reduction target of 2,000 $\mathrm{MMTCO}_{2} \mathrm{e}$, which creates an equilibrium offsets price of between $\$ 38$ and $\$ 47$ per ton. Most projects that are opted in have marginal costs of mitigation well below the equilibrium offsets price, which means that a $\$ 10$ per ton transaction cost will not discourage them from being opted in. If, however, equilibrium offsets prices are much lower, e.g., 15 dollars per ton, doubling the transaction 
cost is likely to have a very significant effect on compliance cost savings from offsets. 
Supplementary Table 22 | Ratio of under-credited emissions reductions to over-credited offsets: Varying offsets supply transaction costs.

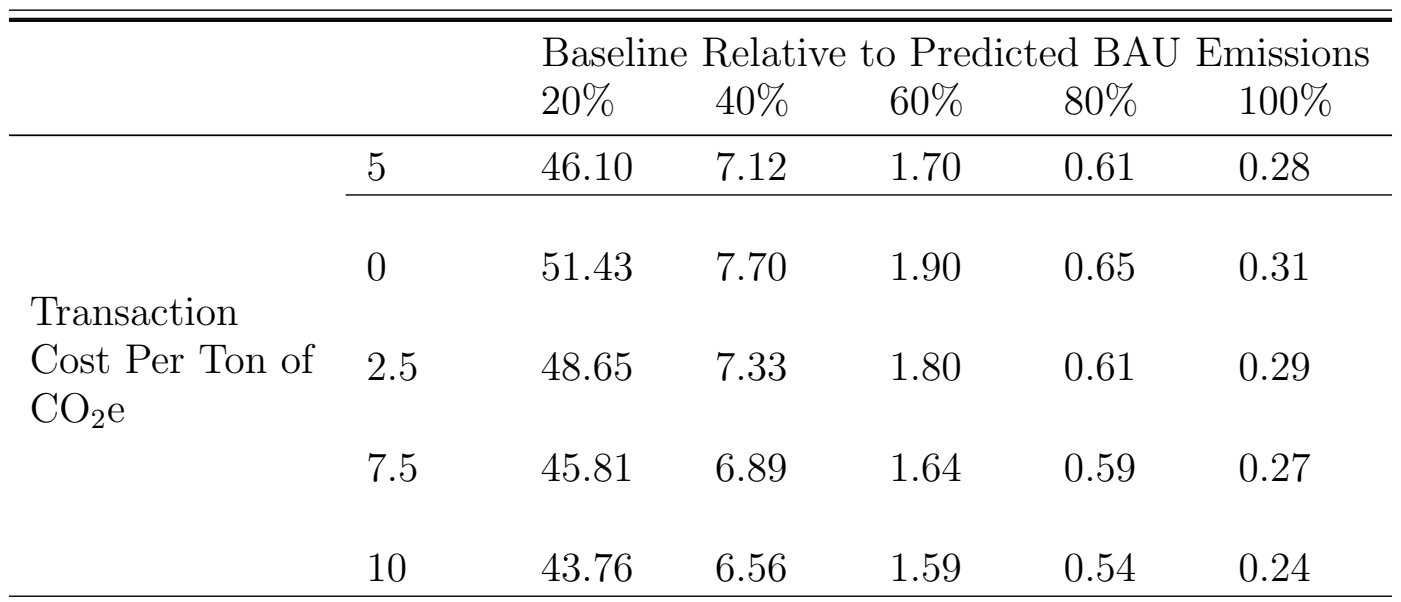

$\overline{\text { A ratio above } 1 \text { implies that the quantity of under-credited emissions reductions }}$ exceeds the supply of over-credited offsets. Transaction costs are denoted in dollars per ton of $\mathrm{CO}_{2} \mathrm{e}$. We assume that transaction costs equal $\$ 5$ per ton of $\mathrm{CO}_{2} \mathrm{e}$ in the benchmark model. 


\section{Supplementary Table 23 | The effect of alternative baseline protocols on offsets supply: Varying the offset supply transaction cost.}

(a) Exact Offsets Supply

\begin{tabular}{ccccc}
\hline \hline & $\begin{array}{c}\text { Predicted } \\
\text { BAU Emissions }\end{array}$ & $\begin{array}{c}\text { Minimize Supply of } \\
\text { Over-Credited Offsets }\end{array}$ & $\begin{array}{c}\text { Maintain Environmental } \\
\text { Integrity }\end{array}$ \\
\hline & 5 & 505 & 338 & 497 \\
\cline { 2 - 5 } & 0 & $+6.53 \%$ & $+5.62 \%$ & $+5.63 \%$ \\
Transaction Cost & & & $+3.25 \%$ & $+2.82 \%$ \\
Per Ton of $\mathrm{CO}_{2} \mathrm{e}$ & 2.5 & $+3.56 \%$ & $-2.96 \%$ & $-3.62 \%$ \\
& 7.5 & $-3.76 \%$ & $-6.51 \%$ & $-7.04 \%$ \\
\hline
\end{tabular}

(b) Total Offsets Supply

\begin{tabular}{ccccc}
\hline \hline & $\begin{array}{c}\text { Predicted } \\
\text { BAU Emissions }\end{array}$ & $\begin{array}{c}\text { Minimize Supply of } \\
\text { Over-Credited Offsets }\end{array}$ & $\begin{array}{c}\text { Maintain Environmental } \\
\text { Integrity }\end{array}$ \\
\hline & 5 & 652 & 338 & 556 \\
\hline & 0 & $+5.06 \%$ & $+5.62 \%$ & $+4.14 \%$ \\
Transaction Cost & 2.5 & $+2.61 \%$ & $+3.25 \%$ & $+1.62 \%$ \\
Per Ton of $\mathrm{CO}_{2} \mathrm{e}$ & & $-2.96 \%$ & $-4.14 \%$ \\
& 7.5 & $-3.22 \%$ & $-6.51 \%$ & $-6.30 \%$ \\
\hline
\end{tabular}

Transaction costs reported in dollars per ton of $\mathrm{CO}_{2} \mathrm{e}$. Exact offsets supply represents the quantity of offsets that corresponds to mitigation from uncapped sector projects. Exact and total offsets supplies are reported in $\mathrm{MMTCO}_{2} \mathrm{e}$ for the benchmark model. The remaining cases are reported relative to the benchmark case. 
Supplementary Table 24 | The effect of alternative baseline protocols on cost savings from including offsets in cap-and-trade programs: Varying the per ton of $\mathrm{CO}_{2}$ transaction cost.

\begin{tabular}{|c|c|c|c|c|}
\hline & & $\begin{array}{c}\text { Predicted } \\
\text { BAU Emissions }\end{array}$ & $\begin{array}{c}\text { Minimize Supply of } \\
\text { Over-Credited Offsets }\end{array}$ & $\begin{array}{c}\text { Maintain Environmental } \\
\text { Integrity }\end{array}$ \\
\hline \multirow{5}{*}{$\begin{array}{l}\text { Transaction } \\
\text { Cost Per Ton } \\
\text { of } \mathrm{CO}_{2} \mathrm{e}\end{array}$} & 5 & 20,394 & 9,849 & 16,872 \\
\hline & 0 & $+16.32 \%$ & $+16.86 \%$ & $+17.27 \%$ \\
\hline & 2.5 & $+8.02 \%$ & $+8.85 \%$ & $+8.33 \%$ \\
\hline & 7.5 & $-7.92 \%$ & $-8.51 \%$ & $-9.30 \%$ \\
\hline & 10 & $-12.30 \%$ & $-17.07 \%$ & $-17.73 \%$ \\
\hline
\end{tabular}

Cost savings are relative to a program that does not allow offsets. These savings are reported in millions of dollars for the benchmark model. The remaining cases are reported relative to the benchmark case. 


\subsection{Transaction Costs as a Fraction of Total Implementation Costs}

In our benchmark model we assume that each project faces a fixed transaction cost equal to $\$ 5$ per ton of $\mathrm{CO}_{2}$ e independent of project characteristics. In this section we relax this assumption by allowing transaction costs to be a function of total implementation costs. There is some evidence that projects with low production costs have low per unit transaction costs (Galik et al., 2012). Here we assume that transaction costs are proportional to total implementation costs, which are defined as transaction costs plus mitigation costs. Previous literature suggests that transaction costs are under 25 percent of total implementation costs (Antinori and Sathaye, 2007; Galik et al., 2012). Therefore we consider the range 0 to 20 percent.

As the fraction of transaction costs increases, the ratio of under-credited emissions reductions to over-credited offsets increases. The ratio increases from 1.88 when there are no transaction costs to 2.55 when transaction costs are 20 percent of total implementation costs with baselines set to 60 percent of predicted BAU emissions. This is because projects that opt in and earn only over-credited offsets have relatively high marginal costs of mitigation. (If they had low marginal costs of mitigation, they would have been opted in and performed mitigation.) These projects are disproportionately burdened by increasing the fraction of total implementation costs that come from transaction costs. As a result, fewer overcredited projects are likely to opt in as the fraction increases. This effect is illustrated in Supplementary Table 26. Even though exact offsets fall as transaction costs increase (Panel (a)), total offsets supply falls much more (Panel (b)), suggesting that a significant quantity of over-credited offsets is no longer supplied.

Supplementary Table 27 reports the sensitivity of cost savings from including offsets in cap-and-trade programs as we vary the fraction of implementation costs that are transaction costs. Increasing the proportion of implementation costs that are transaction costs lowers cost savings from including offsets in cap-and-trade programs. The effect, however, is even more mild than the effect from increasing a flat transaction cost to all projects (Supplementary Table 24). Doubling the transaction cost fraction of total implementation 
costs from 10 percent to 20 percent reduces cost savings by about 2.5 to 5 percent for the different protocols. Cost savings are not very sensitive to increasing the fraction of total implementation costs that are transaction costs because most projects that are opted in have very low implementation costs to begin with. For example, if many projects have marginal costs of mitigation of $\$ 5$ per ton of $\mathrm{CO}_{2}$ or less, then their transaction cost per ton will be under $\$ 1$ per ton of $\mathrm{CO}_{2}$ when the fraction is 20 percent, for a total marginal implementation cost of less than $\$ 5+\$ 1=\$ 6$ dollars per ton of $\mathrm{CO}_{2}$. This increase in implementation costs is relatively small if the equilibrium offsets price is high as it is under a medium capped sector reduction target. 
Supplementary Table 25 | Ratio of under-credited emissions reductions to over-credited offsets: Varying the fraction of implementation costs that are transaction costs.

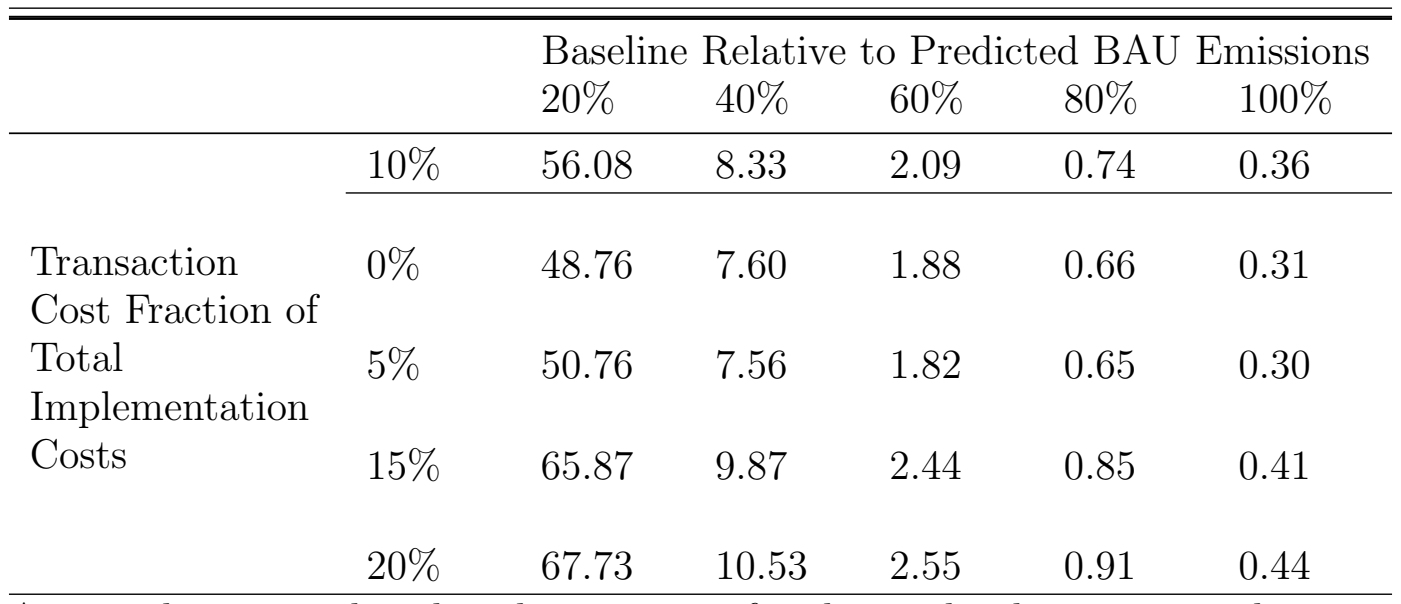

$\overline{\text { A ratio above } 1 \text { implies that the quantity of under-credited emissions reductions }}$ exceeds the supply of over-credited offsets. We assume that transaction costs are a fixed $\$ 5$ per ton of $\mathrm{CO}_{2}$ in the benchmark model. Since we do not model transaction costs as a fraction of total implementation costs in the benchmark model, we use an alternative assumption that transaction costs are 10 percent of total implementation costs to serve as the benchmark. The median transaction cost for the case when transaction costs are 10 percent of total implementation costs is $\$ 5.50$ per ton of $\mathrm{CO}_{2} \mathrm{e}$. 
Supplementary Table 26 | The effect of alternative baseline protocols on offsets supply: Varying the fraction of implementation costs that are transaction costs.

(a) Exact Offsets Supply

\begin{tabular}{lcccc}
\hline & & $\begin{array}{c}\text { Predicted } \\
\text { BAU Emissions }\end{array}$ & $\begin{array}{c}\text { Minimize Supply of } \\
\text { Over-Credited Offsets }\end{array}$ & $\begin{array}{c}\text { Maintain Environmental } \\
\text { Integrity }\end{array}$ \\
\hline & $10 \%$ & 518 & 343 & 506 \\
\cline { 2 - 5 } & $0 \%$ & $+4.05 \%$ & $+4.08 \%$ & $+3.75 \%$ \\
$\begin{array}{l}\text { Transaction Cost } \\
\text { Fraction of Total }\end{array}$ & $5 \%$ & $+1.54 \%$ & $+2.33 \%$ & $+1.78 \%$ \\
$\begin{array}{l}\text { Implementation } \\
\text { Costs }\end{array}$ & $15 \%$ & $-1.54 \%$ & $-1.75 \%$ & $-1.78 \%$ \\
& $20 \%$ & $-4.25 \%$ & $-4.96 \%$ & $-3.36 \%$ \\
\hline
\end{tabular}

(b) Total Offsets Supply

\begin{tabular}{lcccc}
\hline \hline & & $\begin{array}{c}\text { Predicted } \\
\text { BAU Emissions }\end{array}$ & $\begin{array}{c}\text { Minimize Supply of } \\
\text { Over-Credited Offsets }\end{array}$ & $\begin{array}{c}\text { Maintain Environmental } \\
\text { Integrity }\end{array}$ \\
\cline { 2 - 5 } & $10 \%$ & 640 & 343 & 557 \\
\hline $\begin{array}{l}\text { Transaction Cost } \\
\text { Fraction of Total }\end{array}$ & $0 \%$ & $+6.88 \%$ & $+4.08 \%$ & $+4.13 \%$ \\
$\begin{array}{l}\text { Implementation } \\
\text { Costs }\end{array}$ & $5 \%$ & $+5.16 \%$ & $+2.33 \%$ & $+2.15 \%$ \\
& $15 \%$ & $-4.22 \%$ & $-1.75 \%$ & $-1.80 \%$ \\
& $20 \%$ & $-7.66 \%$ & $-4.96 \%$ & $-2.87 \%$ \\
\hline
\end{tabular}

Exact offsets supply represents the quantity of offsets that correspond to mitigation from uncapped sector projects. Exact and total offsets supplies are reported in $\mathrm{MMTCO}_{2} \mathrm{e}$ for the benchmark model. The remaining cases are reported relative to the benchmark case. 
Supplementary Table 27 | The effect of alternative baseline protocols on cost savings from including offsets in cap-and-trade programs: Varying the fraction of implementation costs that are transaction costs.

\begin{tabular}{lcccc}
\hline \hline & & $\begin{array}{c}\text { Predicted } \\
\text { BAU Emissions }\end{array}$ & $\begin{array}{c}\text { Minimize Supply of } \\
\text { Over-Credited Offsets }\end{array}$ & $\begin{array}{c}\text { Maintain Environmental } \\
\text { Integrity }\end{array}$ \\
\cline { 2 - 5 } & $10 \%$ & 20,907 & 11,064 & 17,985 \\
$\begin{array}{l}\text { Transaction } \\
\begin{array}{l}\text { Cost Fraction } \\
\text { of Total }\end{array}\end{array}$ & $0 \%$ & $+13.69 \%$ & $+4.59 \%$ & $+7.79 \%$ \\
$\begin{array}{l}\text { Implementation } \\
\text { Costs }\end{array}$ & $15 \%$ & $-4.84 \%$ & $+2.58 \%$ & $+3.17 \%$ \\
& $20 \%$ & $-8.25 \%$ & $-2.72 \%$ & $-1.92 \%$ \\
\hline
\end{tabular}

Cost savings are relative to a program that does not allow offsets. These savings are reported in millions of dollars for the benchmark model. The remaining cases are reported relative to the benchmark case. 


\section{Project Characteristics}

In this section we describe how the results from the sensitivity analysis can provide guidance to policy makers as they consider which offsets project types to include in cap-and-trade programs. We characterize ten different project types by four key parameters that we vary in the sensitivity analysis. These project types are a relevant subset of the entire universe of offsets project types. We selected them based on their prominence and acceptance in existing cap-and-trade programs (Kile, 2009; Bracmort et al., 2009; RGGI, 2005; Schneider, 2007). This analysis serves as a guide for policy makers considering how to treat different project type with regards to baseline stringency, discounting, and outright banning. Although U.S. federal legislation did not specify guidelines on how to treat different project types and which project types would be allowed in Waxman-Markey, we believe that the projects that we consider are an accurate representation of what project types will be considered in any future

federal climate change mitigation program that has an offsets provision. Supplementary Table 28 summarizes the characteristics of the ten project types considered. 
Supplementary Table 28 | Selected offsets project characteristics.

\begin{tabular}{|c|c|c|c|c|c|}
\hline Offsets Project Type & Program & $\begin{array}{l}\text { BAU Emissions } \\
\text { Uncertainty }\end{array}$ & $\begin{array}{l}\text { Marginal Costs } \\
\text { of Mitigation }\end{array}$ & $\begin{array}{l}\text { Offsets Supply } \\
\text { Potential }\end{array}$ & $\begin{array}{l}\text { Transaction Costs } \\
\text { Per Ton of } \mathrm{CO}_{2}\end{array}$ \\
\hline Landfill methane capture and destruction & RGGI, California & High & Medium & Medium & Medium \\
\hline $\begin{array}{l}\text { Avoided methane emissions from agricultural } \\
\text { manure management operations }\end{array}$ & RGGI, California & Medium & High & Low & High \\
\hline $\begin{array}{l}\text { Reduction in emissions of Sulfur Hexafluoride } \\
\left(\mathrm{SF}_{6}\right) \text { in the electric power sector }\end{array}$ & RGGI & Low & Low & Low & Low \\
\hline Sequestration of carbon due to afforestation & RGGI, California & Medium & Low & High & Low \\
\hline Urban afforestation & California & High & High & Low & Low \\
\hline Hydrofluorocarbon-23 (HFC-23) destruction & $\mathrm{CDM}$ & Low & Low & High & High \\
\hline $\mathrm{N}_{2} \mathrm{O}$ abatement & $\mathrm{CDM}$ & Low & Low & High & Medium \\
\hline Renewable energy & CDM & Medium & Medium & Medium & Low \\
\hline Energy efficiency & RGGI, CDM & Medium/High & Medium & Medium & Medium \\
\hline Avoided deforestation & REDD+ & High & Low & High & Medium/High \\
\hline
\end{tabular}

We give a low, medium or high rating for each project type and characteristic based on data and discussions from various sources. RGGI stands for Regional Greenhouse Gas Initiative. CDM stands for Clean Development Mechanism. REDD+ stands for Reducing Emissions from Deforestation and Forest Degradation.

Landfill methane capture and destruction sources: Bracmort et al. (2009); Antinori and Sathaye (2007); RGGI (2005); Broekhoff and Zyla (2008); EPA (2009b); Avoided methane emissions from agricultural manure management operations sources: Bracmort et al. (2009); Reduction in emissions of Sulfur Hexafluoride $\left(\mathrm{SF}_{6}\right)$ in the electric power sector sources: Antinori and Sathaye (2007); Broekhoff and Zyla (2008); Sequestration of carbon due to afforestation sources: Antinori and Sathaye (2007); Lubowski et al. (2006); EPA (2009b); Urban afforestation sources: RGGI (2005); Hydrofluorocarbon-23 (HFC-23) destruction sources: Wara (2008); $\mathrm{N}_{2} \mathrm{O}$ abatement sources: Wara (2006); Renewable energy sources: Antinori and Sathaye (2007); Energy efficiency sources: Antinori and Sathaye (2007); Broekhoff and Zyla (2008); Avoided deforestation sources: Antinori and Sathaye (2007); Broekhoff and Zyla (2008); Busch et al. (2012) 
Each project type is assigned a qualitative rating for the four key parameters. Our ratings are based on empirical and survey-based studies that we reference next to each project type. We assign a rating for BAU emissions uncertainty, marginal costs of mitigation, offsets supply potential and transaction costs. The ratings are relative to the entire universe of offsets project types. These ratings, however, are averages and may not apply in all settings. For example, studies have found that marginal costs of mitigation for sequestering carbon from afforestation varies considerably across different regions within the United States (Galik et al., 2012; Lubowski et al., 2006; Mason and Plantinga, 2013). Therefore, we suggest that our results be augmented in a future study with a more rigorous disaggregated and datadriven analysis that quantitatively identifies these characteristics for relevant offsets project types.

Our categorization system analyzed with our framework yields some qualitative suggestions for policy makers as they consider including different offsets project types for cost containment purposes. First, several project types that have low marginal costs of mitigation are likely to create more under-credited emissions reductions than over-credited offsets awarded to them (Supplementary Table 7 shows the ratio of under-credited emissions reductions and over-credited offsets as we vary the supply [i.e., marginal cost] of mitigation curve, where a larger supply corresponds to lower marginal costs). This is because potential projects with low marginal costs of mitigation are more likely to opt in when they are assigned a baseline below their BAU emissions, as illustrated with the green area of Figure 1. These project types include HFC-23 destruction, $\mathrm{N}_{2} \mathrm{O}$ abatement, avoided deforestation, and afforestation and $\mathrm{SF}_{6}$ reductions. This rating dimension makes these projects look desirable not only from an environmental standpoint (as under-credited emissions reductions are more likely to cancel over-credited offsets), but also because of economic concerns. Including these projects in cap-and-trade programs can dramatically reduce compliance costs as much cheaper mitigation opportunities are included under the cap (see Supplementary Table 9).

While this result applies to all offsets types, its significance is especially relevant for HFC-23 projects. These project types have recently been banned in the European Union 
Emissions Trading Scheme (EU ETS) for several reasons, including windfall profits and perverse incentives. These projects, however, have been shown to have exceptionally low marginal costs of mitigation (Wara, 2008). Our framework predicts that these projects are likely to opt in to an offsets program even when they are assigned a baseline below their BAU emissions. As a consequence, they are likely to generate large volumes of under-credited emissions reductions as long as they are assigned baselines below their BAU emissions. This result may give policy makers pause before they join the EU ETS action of disallowing HFC-23 offsets from being used for compliance.

Unfortunately, however, many of the projects that have low marginal costs of mitigation and high offsets supply potential also have substantial BAU emissions uncertainty. Supplementary Table 4 suggests that as BAU emissions uncertainty increases, there are relatively more over-credited offsets awarded and relatively fewer under-credited emissions reductions created, having the effect of increasing aggregate emissions. Our framework confirms the standard convention of discounting or banning the use of projects with highly uncertain BAU emissions. Among the project types that have low marginal costs of mitigation, $\mathrm{N}_{2} \mathrm{O}$ abatement and $\mathrm{SF}_{6}$ reductions appear to also have low BAU emissions uncertainty, strengthening the argument for allowing these types of offsets to be used by the capped sector in emissions trading programs.

To protect emissions caps from being compromised and carbon markets from being flooded with over-credited offsets, with a short run reduction target when equilibrium permit and offset prices are low, policy makers may wish to include projects with low BAU emissions uncertainty or to set highly conservative baselines to all projects to avoid awarding projects with too many over-credited offsets. With a high reduction target, however, when carbon prices are expected to be substantially higher as caps are tightened, policy makers should consider relaxing baselines and including potentially risky projects with medium or high BAU emissions uncertainty and low marginal costs of mitigation. 


\section{Supplementary References}

Antinori, C. and J. Sathaye (2007). Assessing transaction costs of project-based greenhouse gas emissions trading. Environmental Energy Technologies Division. Ernest Orlando Lawrence Berkeley National Laboratory.

Baldursson, F. and I. Karatzas (1997). Irreversible investment and industry equilibrium. Finance and Stochastics 1, 69-78.

Baumol, W. J. and W. Oates (1988). The theory of environmental policy. Cambridge University Press.

Bracmort, K., J. Ramseur, J. McCarthy, P. Folger, and D. Marples (2009). Methane capture: Options for greenhouse gas emission reduction. Congressional Budget Office.

Broekhoff, S. and K. Zyla (2008). Outside the cap: Opportunities and limitations of greenhouse gas offsets. World Resources Institute Climate and Energy Policy Series.

Busch, J., R. Lubowski, F. Godoy, M. Steininger, A. Yusuf, K. Austin, J. Hewson, D. Juhn, M. Farid, and F. Boltz (2012). Structuring economic incentives to reduce emissions from deforestation within Indonesia. Proceedings of the National Academy of Sciences 109(4), 1062-1067.

Bushnell, J. (2012). The economics of carbon offsets. National Bureau of Economic Research.

EPA (2009a). Processed marginal abatement cost (MAC) curves.

EPA (2009b). Updated forestry and agriculture marginal abatement cost curves. http://www.epa.gov/climatechange/economics/downloads/EPAactivities/ HR2454Analysis-DataAnnex.zip.

EPA (2010). Environmental protection agency. data annex coverage and caps: Emissions inventory. scenario 7. http://www.epa.gov/climatechange/economics/downloads/ EPAactivities/HR2454Analysis-DataAnnex.zip.

Fell, H., D. Burtraw, R. Morgenstern, and K. Palmer (2012). Soft and hard price collars in a cap-and-trade system: A comparative analysis. Journal of Environmental Economics and Management 64(2), 183-198.

Fell, H. and R. Morgenstern (2010). Alternative approaches to cost containment in a capand-trade system. Environmental and Resource Economics 47, 275-297.

Galik, C., D. Cooley, and J. Baker (2012). Analysis of the production and transaction costs of forest carbon offset projects in the USA. Journal of Environmental Management 112, $128-136$.

Kile, J. (2009). The use of agricultural offsets to reduce greenhouse gases. Congressional Budget Office Testimony.

Lubowski, R., A. Plantinga, and R. Stavins (2006). Land-use change and carbon sinks: Econometric estimation of the carbon sequestration supply function. Journal of Environmental Economics and Management 51, 135-152. 
Lucas, R. and E. Prescott (1971). Investment under uncertainty. Econometrica 39, 659-781.

Mason, C. and A. Plantinga (2013). The additionality problem with offsets: Optimal contracts for carbon sequestration in forests. Journal of Environmental Economics and Management 66, 1-14.

RGGI (2005). Regional greenhouse gas initiative. offsets: IPM modeling assumptions.

Schneider, L. (2007). Is the CDM fulfilling its environmental and sustainable development objectives? An evaluation of the CDM and options for improvement. Oko-Institut: Institute for Applied Ecology.

Wara, M. (2006). Measuring the clean development mechanism's performance and potential. Stanford University Program on Energy and Sustainable Development Working Paper Number 56 .

Wara, M. (2008). Measuring the clean development mechanism's performance and potential. UCLA Law Review 55, 1759-1803. 